\title{
Feasibility of Electron Cooling for Low-Energy RHIC Operation
}

A. Fedotov, I. Ben-Zvi, X. Chang, D. Kayran, V. Litvinenko, E. Pozdeyev and T. Satogata

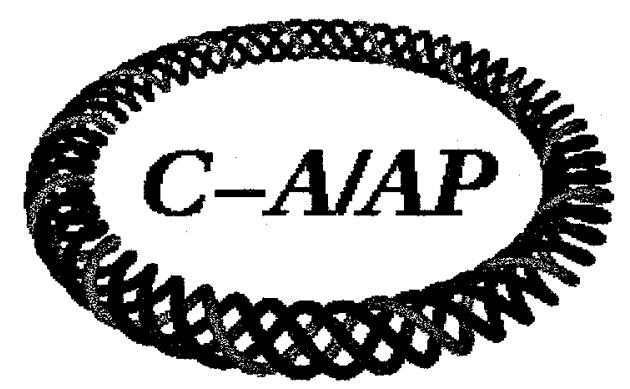

\section{Collider-Accelerator Department Brookhaven National Laboratory Upton, NY 11973}

Notice: This document has been authorized by employees of Brookhaven Science Associates, LLC under Contract No. DEAC02-98CH10886 with the U.S. Department of Energy. The United States Government retains a non-exclusive, paid-up, irrevocable, world-wide license to publish or reproduce the published form of this document, or allow others to do so, for United States Government purposes. 


\title{
FEASIBILITY OF ELECTRON COOLING FOR LOW-ENERGY RHIC OPERATION
}

\author{
A. Fedotov, I. Ben-Zvi, X. Chang, D. Kayran, V. Litvinenko, E. Pozdeyev and T. Satogata \\ Collider-Accelerator Department, BNL
}

A concrete interest in running RHIC at low energies in a range of $2.5-25 \mathrm{GeV} /$ nucleon total energy of a single beam has recently emerged. Providing collisions in this energy range, which in the RHIC case is termed "low-energy" operation, will help to answer one of the key questions in the field of QCD about existence and location of a critical point on the QCD phase diagram. However, luminosity projections are relatively low for the lowest energy points of interest. Luminosity improvement can be provided with electron cooling applied directly in RHIC at low energies. This report summarizes the expected luminosity improvement with electron cooling, possible technical approaches and various limitations.

Table of Contents

1. Overview

2. Expected Performance

3. Luminosity limitations

4. Luminosity with Electron Cooling

5. Considerations for Electron Cooler

$5.1 \mathrm{DC}$ cooler

$5.256 \mathrm{MHz}$ RF gun cooler

$5.3703 \mathrm{MHz}$ RF gun cooler

5.4 Non-magnetized vs. magnetized approach

5.5 Pros and Cons of various approaches

6. Cost estimate

7. Acknowledgements

8. References

Appendix:

A1. List of parameters

A2. Parameters of DC electron beam

A3. Electron beam dynamics for $56 \mathrm{MHz}$ RF gun approach

A4. Electron beam dynamics for $703 \mathrm{MHz}$ RF gun approach

A5. Cooling section 


\section{OVERVIEW}

Recently, a strong interest emerged in running the Relativistic Heavy Ion Collider (RHIC) at low beam total energies of 2.5-25 GeV/nucleon [1-3], substantially lower than the nominal beam total energy of $100 \mathrm{GeV} /$ nucleon. Collisions in this low energy range are motivated by one of the key questions of quantum chromodynamics (QCD) about the existence and location of critical point on the QCD phase diagram [4].

RHIC data will complement existing fixed-target data from AGS and SPS. In this energy range an energy scan will be conducted over about 7 different energies. There are several challenges to the operation of RHIC at such low energies. To evaluate the severity of these challenges and make projections for low-energy operation there have been several short test runs during RHIC operations in 2006, 2007 and 2008. Results of these test runs are summarized in Refs. [5].

\section{EXPECTED PERFORMANCE}

The beam lifetime observed during the test runs was clearly limited by machine nonlinearities. This performance can be improved provided sufficient time is given for machine development at these low energies. After the lifetime caused by nonlinearities is improved the strongest limitation comes from transverse and longitudinal Intra-beam Scattering (IBS), and ultimately by the space-charge limit.

Examples of stores at intermediate energy point with $\gamma=4.9$ are shown in Figs. 2.1 and 2.2. During the first test run in June 2007 the lifetime in the machine was very short and dominated by nonlinearities, as shown in Fig. 2.1. These nonlinearities were intentionally strongly driven during this test run to stabilize against observed head-tail instabilities. During the latest test run in March 2008 the lifetime was improved with the help of changes to defocusing sextupole configuration, as can be seen in Fig. 2.2. The store length was extended from 15 minutes in 2007 to 1 hour in 2008. 


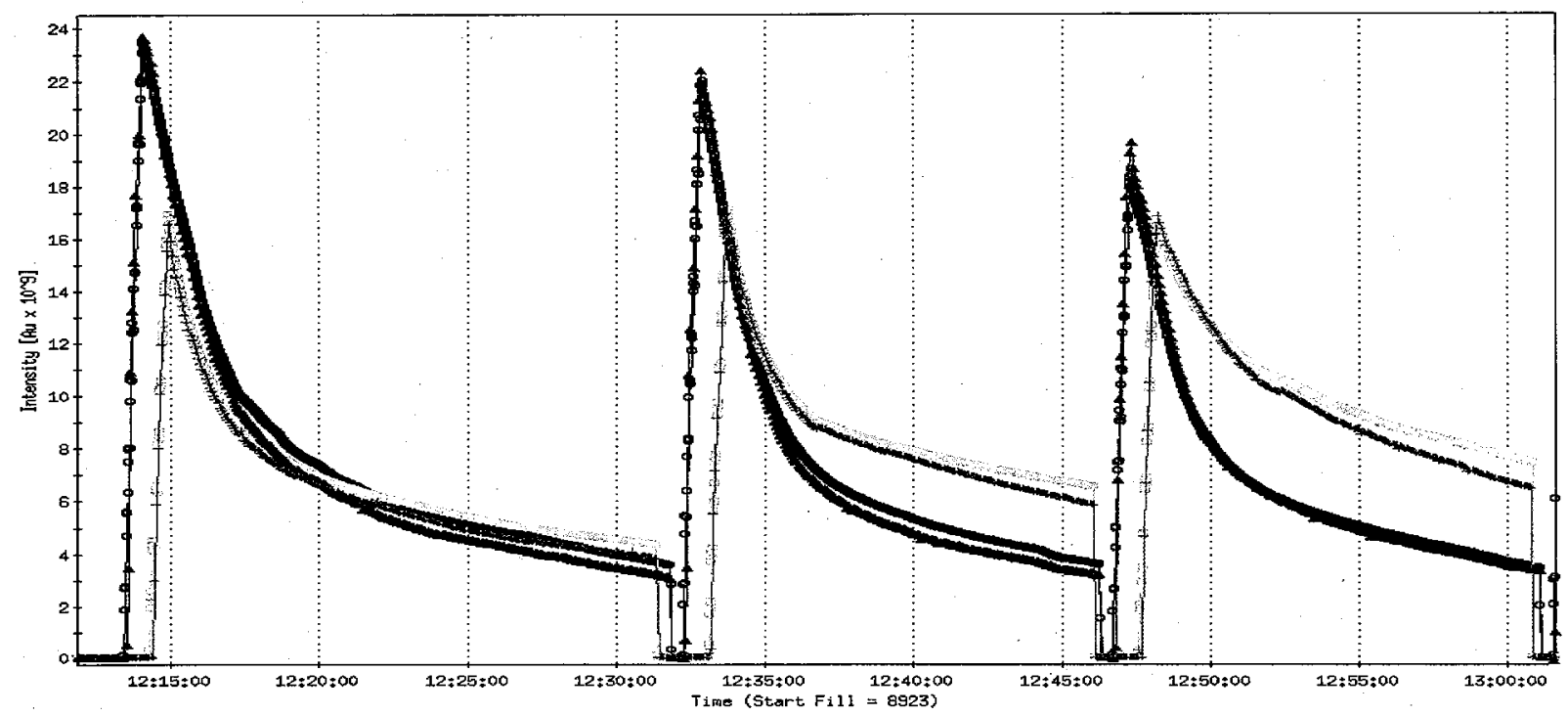

Fig. 2.1. Typical stores during June 2007 test run with $\mathrm{Au}$ ions at $\gamma=4.9$. 1) Blue ring: upper blue curve - total DCCT signal; lower light blue curve - bunched beam intensity with WCM. 2) Yellow ring: upper yellow curve - total DCCT signal; lower orange curve - bunched beam intensity with WCM.

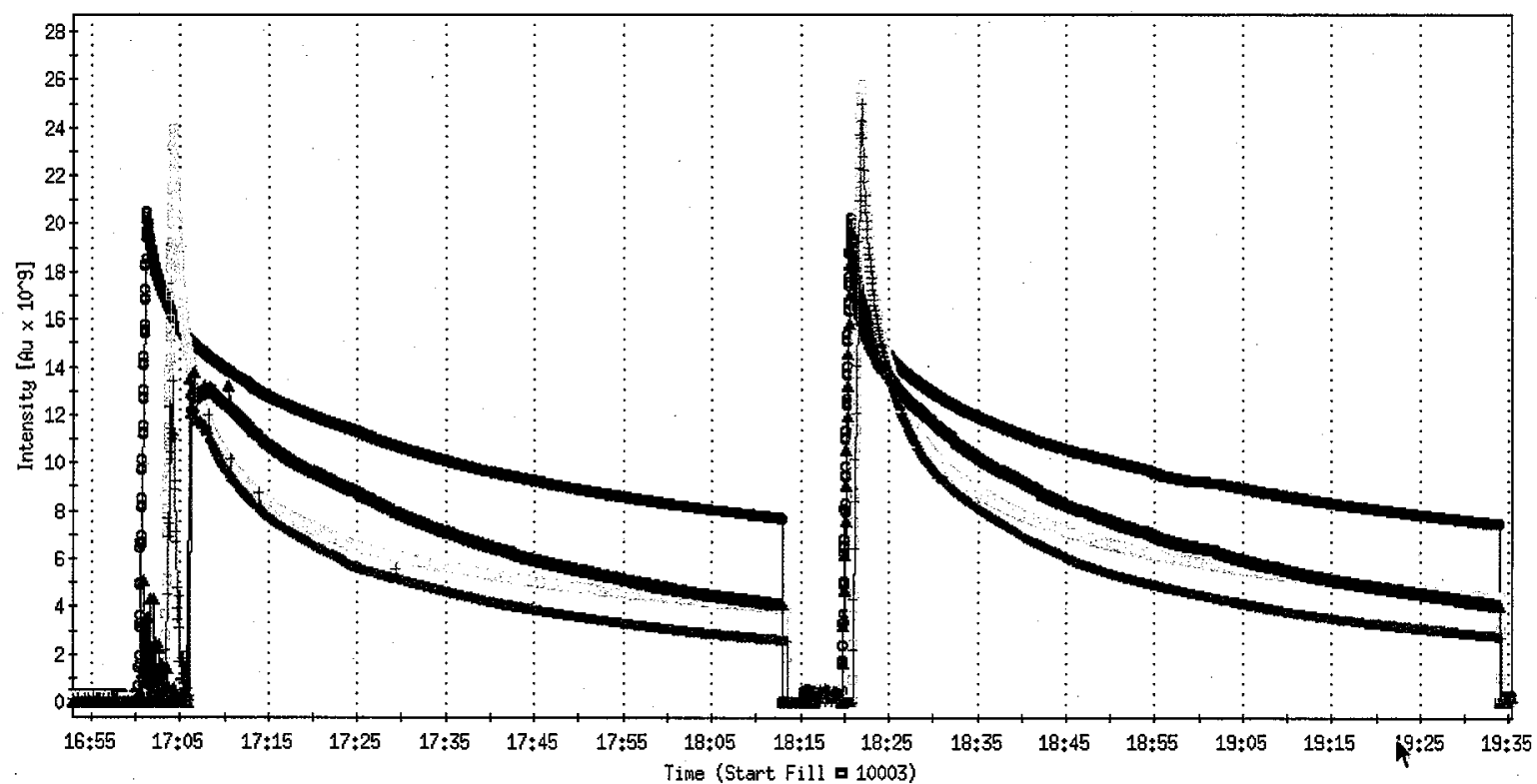

Fig. 2.2. Typical two stores during March 11, 2008 test run with $\mathrm{Au}$ at $\gamma=4.9 .1$ ) Blue ring: upper blue curve - total DCCT signal; lower light blue curve - bunched beam intensity with WCM. 2) Yellow ring: upper yellow curve - total DCCT signal; lower orange curve - bunched beam intensity with WCM. 
For the test run in March 2008, the injected longitudinal emittance of gold ion bunches was close to the RF bucket acceptance. As a result, one can see significant loss from the RF bucket (lower light blue curve for Blue ring and orange curve for Yellow ring) driven by longitudinal IBS in Fig. 2.2. The total RF voltage was $430 \mathrm{kV}$ and $500 \mathrm{kV}$ for the Blue and Yellow rings, respectively.

Presently, the uncertainty in the expected useful luminosity at low energies is very large. For example, for the test run performed on March 11, 2008 the reported rate of beam-beam counter coincidence signals was about $340 \mathrm{~Hz}$. However, the rate of useful physics events is estimated to be only about $1 \mathrm{~Hz}$. The reason for such a small number of useful events is presently under study. Some improvements in the useful luminosity are straightforward. For example, $1 \mathrm{~Hz}$ can be doubled by doubling the number of bunches to 108 . Since the test run machine was clearly limited by nonlinearities, some improvement in machine performance is also expected with additional machine development time. We expect that an additional factor of 3 may be possible at energies around $\gamma=4.9$. As a result, the rate of useful events near $\gamma=4.9$ may be increased from $1 \mathrm{~Hz}$ to about $6 \mathrm{~Hz}$, with future machine development improvements. Table 2.1 shows scaling to other energies which includes the discussed improvements at low energies.

\begin{tabular}{|c|c|c|c|c|c|}
\hline $\begin{array}{l}{ }_{\mathrm{s}_{\mathrm{NN}}} \mathrm{GeV} \text { (center of mass } \\
\text { energy) }\end{array}$ & 5 & 6.3 & 7.6 & 8.8 & 12.3 \\
\hline$\gamma$ & 2.7 & 3.7 & 4.1 & 4.7 & 6.6 \\
\hline $\begin{array}{l}\text { Estimated rate of useful } \\
\text { events, } \mathrm{Hz}\end{array}$ & 0.5 & 2.0 & 4.0 & 6.0 & 15 \\
\hline $\begin{array}{l}\text { Days needs to accumulate } \\
5 \mathrm{M} \text { useful events }\end{array}$ & 143 & 36 & 18 & 12 & 5 \\
\hline $\begin{array}{l}\text { Expected improvement } \\
\text { factor from cooling }\end{array}$ & 3 & 3 & 3 & 4 & $5-6$ \\
\hline $\begin{array}{l}\text { Days needed to accumulate } \\
5 \mathrm{M} \text { useful events with } \\
\text { electron cooling }\end{array}$ & 48 & 12 & 6 & 3 & 1 \\
\hline
\end{tabular}

Table 2.1 Estimates based on an assumption of $6 \mathrm{~Hz}$ useful events (after expected machine improvements in the future) at $\gamma=4.9$ with $80 \%$ of calendar time spent in physics.

It is clear that the first improvement in luminosity should come from improvement of lifetime due to nonlinearities. In addition, both transverse and longitudinal IBS growth can be compensated by electron cooling technique [6]. Unfortunately, the ultimate limitation due to space charge prohibits strong cooling at the lowest energy points, which would give an otherwise dramatic increase in the luminosity.

Applying electron cooling directly in RHIC will increase the average integrated luminosity significantly, and will provide long stores for physics. With electron cooling it seems feasible to have about a factor of 3-6 improvement in average luminosity depending on the energy (see Sections 3 and 4 for details), which would enable detailed studies of signatures of the Critical Point.

Note that a limited factor of 3 improvement from electron cooling for lowest energy points in Table 2.1 is driven by the space-charge limit (see Sections 3-4) and assumes that machine performance will be substantially improved without cooling (no strong intensity drop in the first few minutes, as can be seen in Fig. 2.2). If, however, significant fast initial intensity drop remains, then spacecharge limit is relaxed, and electron cooling can provide additional factors of improvements on top 
of the factor of 3 given in Table 2.1 due to a possibility of cooling ion beam emittance with a subsequent reduction of beta function at the Interaction Point.

In addition, if signatures of the Critical Point are found, it is expected that a request for high statistics, for example 50M events, will follow. Electron cooling in RHIC enables acquisition of such statistics in a reasonable period of time. Without electron cooling, acquisition of high statistics of about 50M events is impractical.

\section{LUMINOSITY LIMITATIONS}

\section{$3.1 \mathrm{IBS}$}

One of the major effects which leads to the luminosity decrease during a store cycle is intrabeam scattering (IBS) inside each bunch of both beams circulating in the rings. IBS leads to an increase of the bunch length and of the transverse beam emittance. The increase of bunch length due to IBS results in beam loss from the RF bucket. This is shown in Figs. 3.1-3.2 (simulations of ion beam dynamics presented in this document are done using BETACOOL code [7]). Simulations are shown for ion beam parameters in Table 3.1. At these low energies, strong IBS growth rates can be easily counteracted with electron cooling.

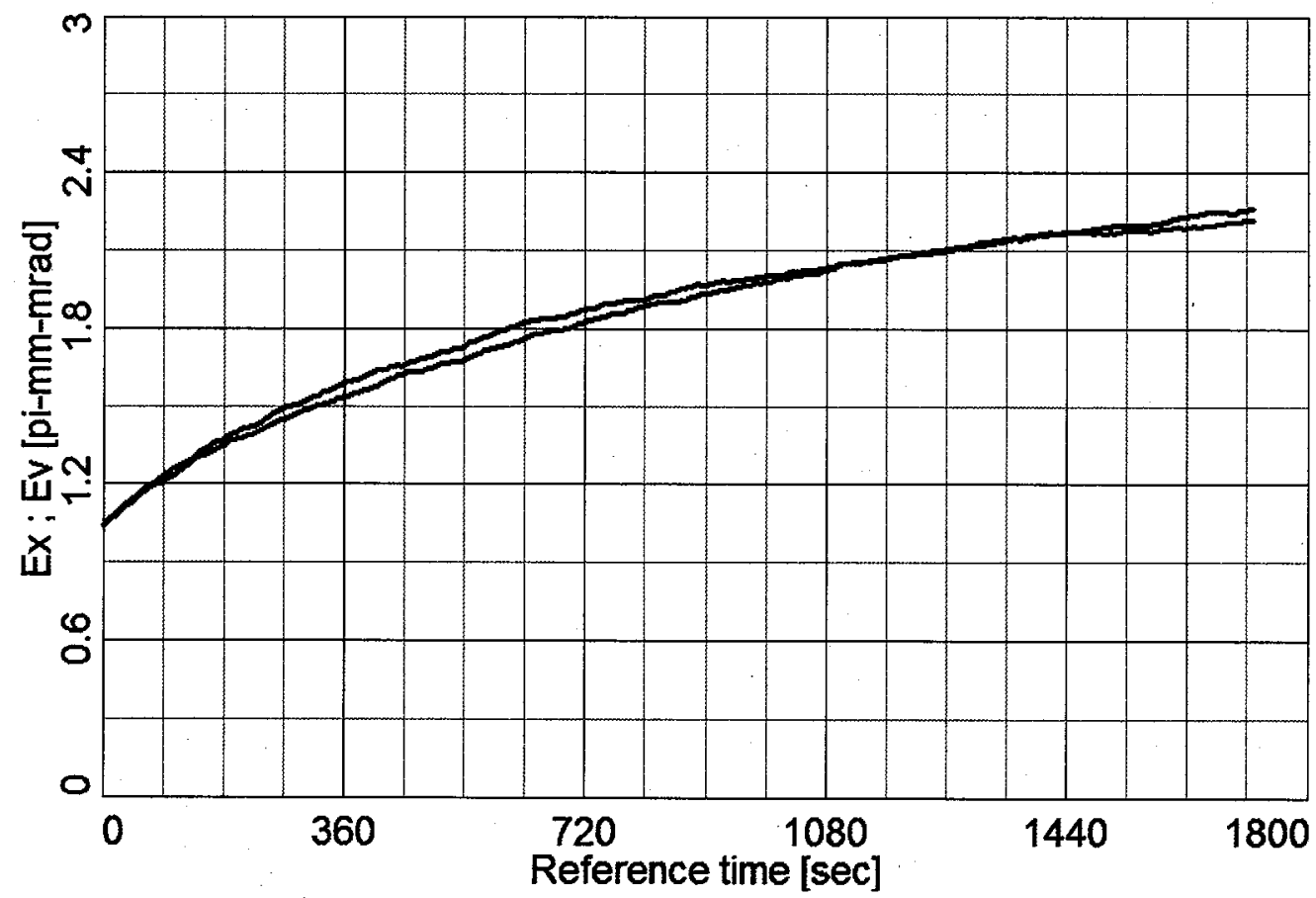

Fig. 3.1. Simulated growth of rms unnormalized emittance due to IBS at $\gamma=2.67$. Red - horizontal emittance. Blue - vertical emittance. 


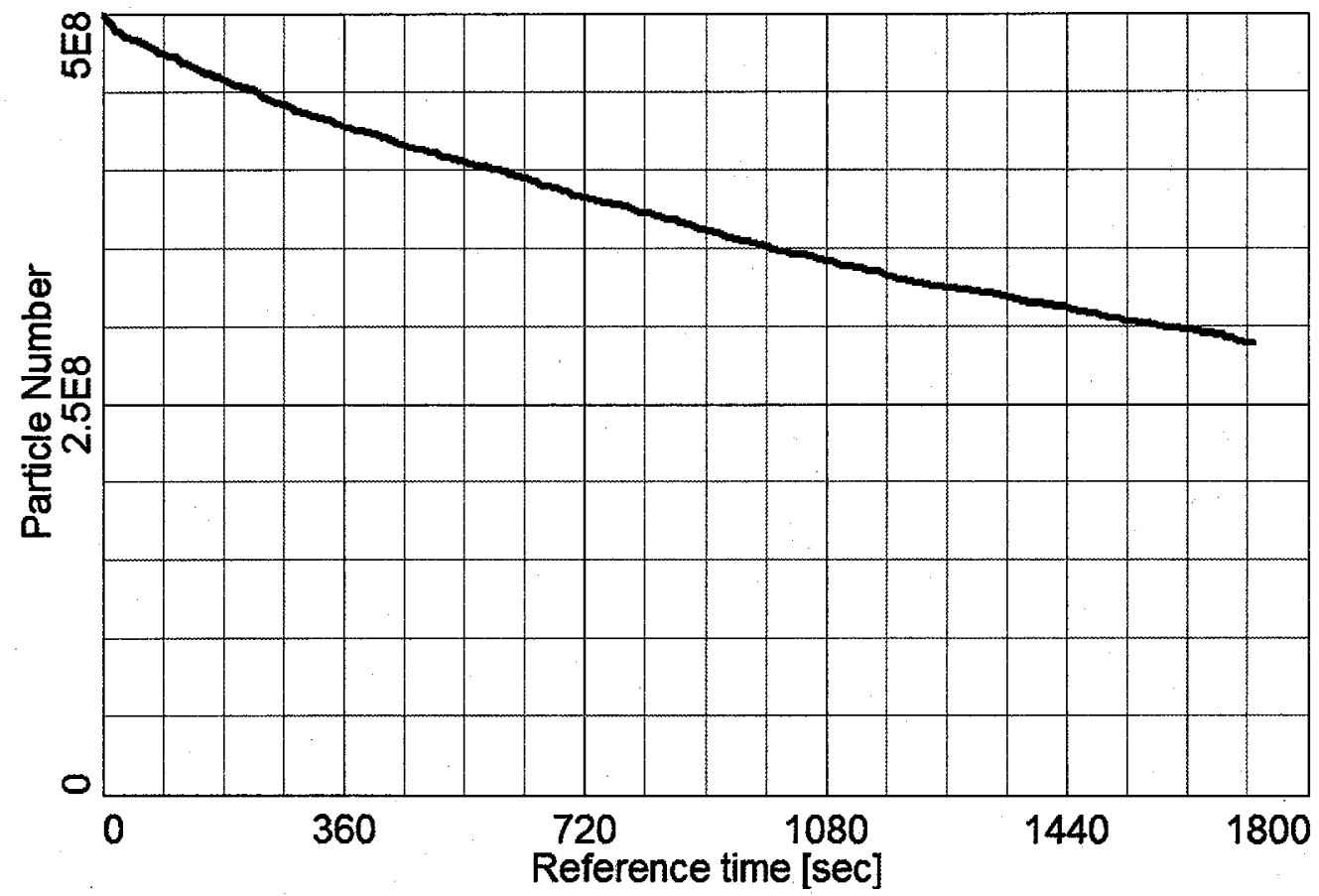

Fig. 3.2. Simulated decrease of bunch intensity due to loss from RF bucket caused by longitudinal IBS at $\gamma=2.67$.

\begin{tabular}{|l|l|}
\hline$\gamma$ & 2.7 \\
\hline Number of ions per bunch, $10^{9}$ & 0.5 \\
\hline $\begin{array}{l}\text { Initial transverse 95\% normalized emittance, } \\
\text { mm mrad }\end{array}$ & 15 \\
\hline rms momentum spread & 0.0005 \\
\hline RF harmonic & 387 \\
\hline
\end{tabular}

Table 3.1. Parameters of ion beam used in IBS simulations for Figs. 3.1-3.2.

\subsection{Space-charge tune shift}

For a Gaussian transverse distribution, the maximum incoherent space-charge tune shift can be estimated using the following formula:

$$
\Delta Q=-\frac{Z^{2} r_{p}}{A} \frac{N_{i}}{4 \pi \beta^{2} \gamma^{3} \varepsilon} \frac{F_{c}}{B_{f}},
$$

where $F_{c}$ is a form factor which includes correction coefficients due to beam pipe image forces (the Laslett coefficients), $N_{\mathrm{i}}$ is the number of ions per bunch, $\varepsilon$ is the unnormalized rms emittance and $B_{f}$ is the bunching factor (mean/peak line density). Here, for simplicity, we assume $F_{c}=1$, thus 
considering only self-fields of the beam. It is also correct assumption for vacuum chamber of circular cross section, for which incoherent electric image coefficients vanish due to symmetry.

For the low energy points in RHIC, the RF bucket acceptance is relatively small due to limited RF voltage. The longitudinal emittance of the incoming ion beam is comparable to or bigger than the RF bucket acceptance. As a result, the RF bucket is completely filled after injection. For the estimate of space-charge tune in such a case, we assume a full bucket with a parabolic ion beam profile, using notation $\Delta \mathrm{Q}_{\mathrm{fb}}$.

\begin{tabular}{|l|l|l|l|l|l|}
\hline$\gamma$ & $\begin{array}{l}\mathrm{h} \\
\text { (harmonic } \\
\text { number) }\end{array}$ & $\begin{array}{l}\varepsilon_{95 \%, \mathrm{n}} \\
\mu \mathrm{m}\end{array}$ & $\begin{array}{l}\mathrm{N}_{\mathrm{i}} \times 10^{9} \\
\text { (intensity per } \\
\text { bunch) }\end{array}$ & $\begin{array}{l}\Delta \mathrm{Q}_{\mathrm{G}} \\
\text { (space charge } \\
\text { tune shift for } \\
\text { Gaussian } \\
\text { longitudinal } \\
\text { profile) }\end{array}$ & $\begin{array}{l}\Delta \mathrm{Q}_{\mathrm{fb}} \\
\text { (full bucket, } \\
\text { parabolic } \\
\text { longitudinal } \\
\text { profile) }\end{array}$ \\
\hline 2.67 & 387 & 15 & 0.5 & 0.12 & 0.07 \\
\hline 3.37 & 375 & 15 & 1 & 0.15 & 0.08 \\
\hline 4.41 & 369 & 15 & 1 & 0.1 & 0.05 \\
\hline 4.7 & 366 & 15 & 1 & 0.07 & 0.04 \\
\hline 6.6 & 360 & 15 & 1 & 0.04 & 0.02 \\
\hline
\end{tabular}

Table 3.2. Incoherent space-charge tune shifts for different energy points.

Note that one gets a higher luminosity for low-energy points if rather than decreasing the intensity per bunch one increases the emittance keeping the intensity constant and staying at the space-charge limit. However, for the RHIC case, the increase in the emittance is limited by the beam size in the triplets, which results in a beam loss.

\subsection{Beam-beam parameter}

The linear part of the tune shift due to interaction with a colliding bunch is called the "beam-beam" parameter. If the beam-beam parameter exceeds some limiting value one can have significant increase of emittance due to diffusion process. In hadron machines typical limiting value for the beam-beam parameter per single Interaction Point (IP) is 0.005-0.01.

For a round beam, the beam-beam parameter is:

$$
\xi=\frac{Z^{2} r_{p}}{A} \frac{N_{i}}{4 \pi \beta^{2} \gamma \varepsilon} \frac{1+\beta^{2}}{2},
$$

where $r_{p}$ is the proton classical radius. 
For round beams, the peak luminosity can be calculated according to the following formula:

$$
L=\frac{N_{i}^{2}}{4 \pi \varepsilon \beta^{*}} F_{\text {coll }} f\left(\frac{\sigma_{s}}{\beta^{*}}\right)
$$

where $\mathrm{N}_{\mathrm{i}}$ is the ion number per bunch, $\varepsilon$ is the transverse unnormalized rms emittance, $\beta^{*}$ is the beta function in the IP and $\sigma_{s}$ is the rms value of the longitudinal beam size. $F_{\text {coll }}$ is the collision repetition frequency, which equals to the bunch revolution frequency $\mathrm{F}_{\text {rev }}$ multiplied by the bunch number $\mathrm{N}_{\text {bunches. }}$. The factor $f$ (the "hourglass effect") is defined by the formula

$$
f\left(\frac{\sigma_{s}}{\beta^{*}}\right)=\frac{1}{\sqrt{\pi}} \int_{-\infty}^{\infty} \frac{\exp \left(-u^{2}\right) d u}{\left.1+\left(\frac{u \sigma_{s}}{\beta^{*}}\right)^{2}\right]} .
$$

This factor is close to unity when the longitudinal rms beam size is much less than the value of beta function at the IP, and decreases when $\sigma_{\mathrm{s}}$ is increased. For low-energy RHIC operation we presently use $\beta^{*}=10 \mathrm{~m}$, which is much bigger than rms bunch length, so that we can neglect this hourglass factor in the estimates.

If the single bunch luminosity is limited by the beam-beam effect it can be expressed via $\xi$ as:

$$
L=\frac{A}{Z^{2} r_{p}} \frac{N_{i} c}{\beta^{*} C} \frac{2 \gamma \beta^{2}}{1+\beta^{2}} f\left(\frac{\sigma_{s}}{\beta^{*}}\right) \xi,
$$

where $\mathrm{C}$ is the ring circumference.

If the luminosity is limited by the space-charge tune shift value $\Delta \mathrm{Q}$, then it can be expressed as:

$$
L=\frac{A}{Z^{2} r_{p}} \frac{N_{i} c}{\beta^{*}} \frac{\sqrt{2 \pi} \sigma_{s}}{C^{2}} \gamma^{3} \beta^{2} f\left(\frac{\sigma_{s}}{\beta^{*}}\right) \Delta Q .
$$

In the general case, the luminosity is limited by a minimum value from either Eq. (3.5) or (3.6). For the RHIC parameters, the single bunch luminosity is plotted in Figs. 4.1-4.2 as a function of beam energy for fixed values of $\Delta Q=0.05$ and $\xi=0.005$. 


\section{LUMINOSITY WITH COOLING}

In Ref. [1] the proposed list of collision energies for the QCD critical point search corresponds to ion beam kinetic energies of $\mathrm{E}_{\mathrm{k}, \mathrm{i}}=1.6,2.2,2.9,3.45,5.2,8.1$ and $13.1 \mathrm{GeV} /$ nucleon. For higher points from this energy range (8-13 GeV/nucleon), the luminosity is expected to be relatively large. However, for the lowest energy points, the expected luminosities are low. The luminosity can be improved by providing electron cooling of ion bunches. To apply electron cooling for ion beam kinetic energies of $E_{k, i}=1.6,2.2,2.9,3.45,5.2 \mathrm{GeV} /$ nucleon, requires electron beam with a kinetic energy range of $0.86-2.8 \mathrm{MeV}$.

Electron cooling can easily counteract IBS at such low energies. If IBS would be the only limitation, one could achieve small hadron beam emittance and bunch length with the help of electron cooling, resulting in a dramatic luminosity increase [8-9]. Unfortunately, at lowest energy points in RHIC the defining limitation is expected to be due to the space charge.

When the space-charge tune shift becomes significant, the beam overlaps many machine imperfection resonances, leading to large beam losses and poor lifetime. For machines where beam spends only tens of msec in high space-charge regime, as well as one compensates machine resonances, the tolerable space-charge tune shift can be as big as $\Delta \mathrm{Q}=0.2-0.5$. However, for a long storage time, acceptable tune shifts are much smaller. In some machines, lifetimes of few minutes were achieved with tune shifts of about 0.1 or higher [10]. In LEAR, it was possible to accommodate space-charge tune shifts of about 0.1 , with a proper choice of working point and electron cooling [11]. For RHIC, we are interested in lifetimes much longer than a few minutes. As a result, we take space-charge tune shift values of about 0.05 as a limit for our present estimates.

Table 3.2 shows that in order not to exceed such space-charge limit for lowest energy point with $\gamma=2.67$ one cannot decrease emittance (with cooling) to lower values. In fact, for $95 \%$ normalized emittance of $15 \mathrm{~mm}$ mrad even intensity of ion bunch has to be reduced to $0.5 \times 10^{9}$ to have an acceptable space-charge tune shift. Note that one can get higher luminosities for low-energy points if rather than decreasing the intensity per bunch one increases emittance keeping intensity constant and staying at the space-charge limit, as shown in Fig. 4.1. As a result, the role of electron cooling at the lowest energy points becomes just to counteract IBS which will prevent transverse emittance growth and intensity loss from the bucket due to longitudinal IBS, resulting in long stores with constant luminosity.

For RHIC parameters with $\beta^{*}=10 \mathrm{~m}$, the single bunch luminosity limited by the space-charge tune shift of $\Delta \mathrm{Q}=0.05$ (for the plot $\mathrm{N}_{\mathrm{i}}=1 \times 10^{9}$ was used for all energy points) is shown in Fig. 4.1. One can see a strong cubic dependence of the maximum achievable luminosity on energy. It also shows what values of luminosity can be reached when the emittance of the beam is decreased through electron cooling to the space-charge limit of $\Delta Q=0.05$. Note, that the single-bunch luminosity values shown in Fig. 4.1 are ideal maximum projections, which may be reached in a machine in the absence of other limitations. For example, in a 1-day test run on March 11, 2008 recorded singlebunch luminosities were about factor of 10 smaller than in Figs. 4.1-4.2, showing that there is still some room for luminosity improvement.

For higher energy points, in addition to counteracting IBS electron cooling provides emittance decrease needed to operate at the space-charge limit. This, in turn, allows us to reduce beta function 
at the Interaction Point. As a result, electron cooling provides a larger luminosity gain for higher energy points.

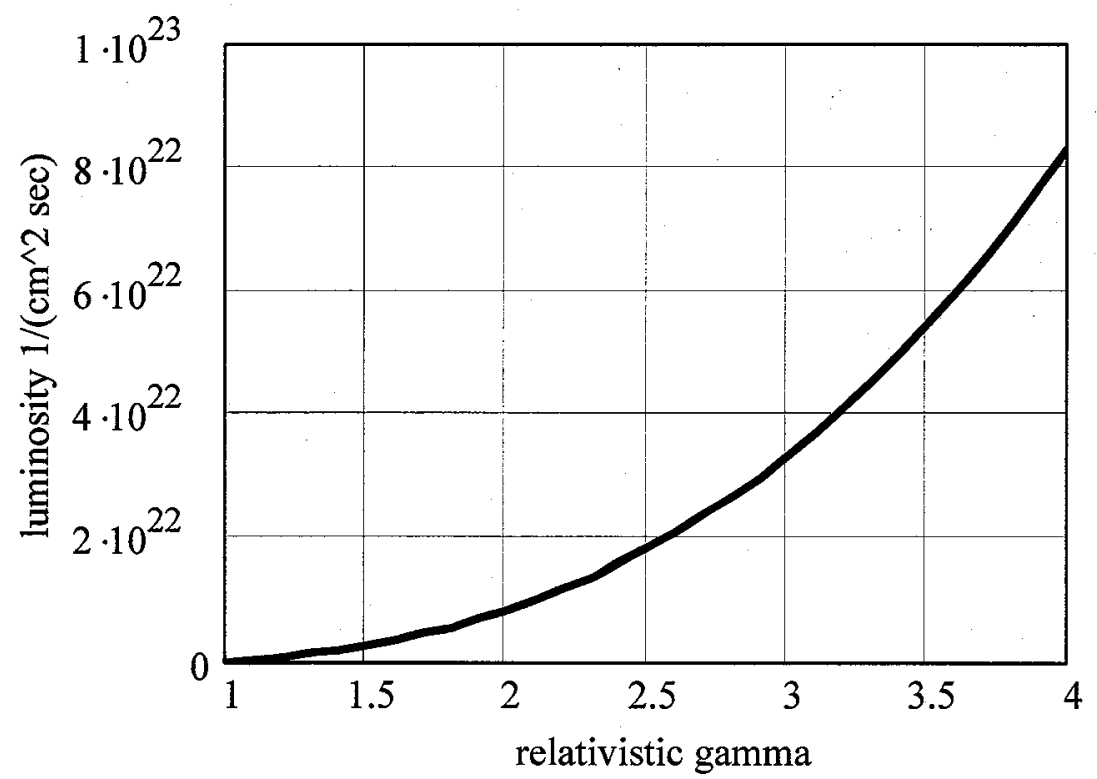

Fig. 4.1. Single-bunch luminosity limitation caused by space-charge limit with $\Delta \mathrm{Q}=0.05$.

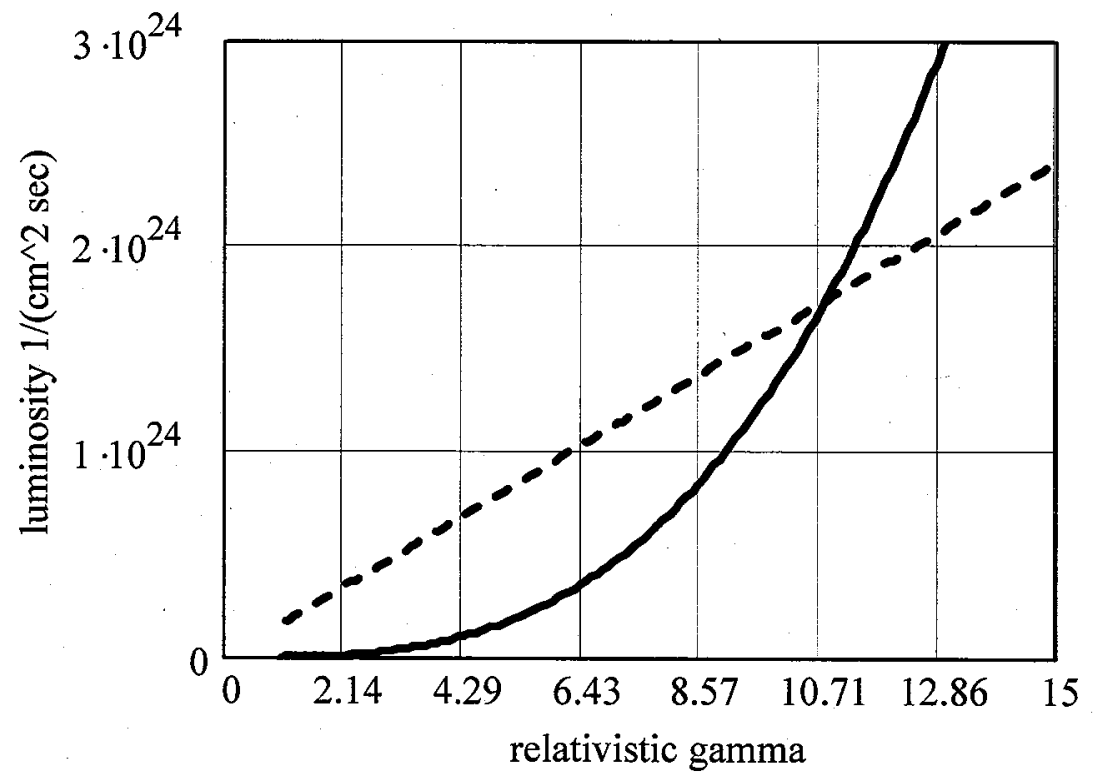

Fig. 4.2. Single-bunch luminosity limitation 1) red line - by space-charge with $\Delta Q=0.05$ 2) blue dash line - by beam-beam parameter with 0.005 per IP.

Figure 4.2 shows the limits for single bunch luminosity due to space charge with $\Delta Q=0.05$ (red line) or beam-beam with $\xi=0.005$ (blue dash line). One can see that below $\gamma=10.7$ the maximum achievable single bunch luminosity is limited by space charge while above $\gamma=10.7$ it is limited by beam-beam effect. 
Figure 4.3 shows simulation of luminosity performance with and without electron cooling for $\gamma=2.67$. Simulations are done for ion bunch intensity $\mathrm{N}_{\mathrm{i}}=0.5 \times 10^{9}$, initial $95 \%$ normalized emittance of $15 \mathrm{~mm}$ mrad, rms momentum spread $\sigma_{\mathrm{p}}=5 \times 10^{-4}$, rms bunch length $\sigma_{\mathrm{s}}=1.9 \mathrm{~m}$, and 56 bunches. As a result of longitudinal IBS and particle loss from RF bucket, there is an intensity loss. Also there is still significant emittance increase due to transverse IBS even for reduced bunch intensity of $\mathrm{N}_{\mathrm{i}}=0.5 \times 10^{9}$ per bunch. This results in a rapid luminosity drop shown in Fig. 4.3 with black circles. The resulting store length becomes relatively short - one has to refill machine every 10-15 minutes. With electron cooling, the transverse emittance was kept constant, as well as longitudinal IBS was counteracted. As a result, electron cooling provided long store time with relatively constant luminosity. Overall gain in luminosity with electron cooling, taking into account needed time for refill between short stores without cooling, is about factor of 3 in average luminosity. Larger luminosity gains might be possible if one could operate with space-charge tune shifts larger than $\Delta \mathrm{Q}=0.05$ (operation with slightly larger tune shifts may be expected with the help of cooling). Note, that these simulations do not include beam lifetime associated with nonlinearities of the machine, and thus show "ideal" performance of the machine.

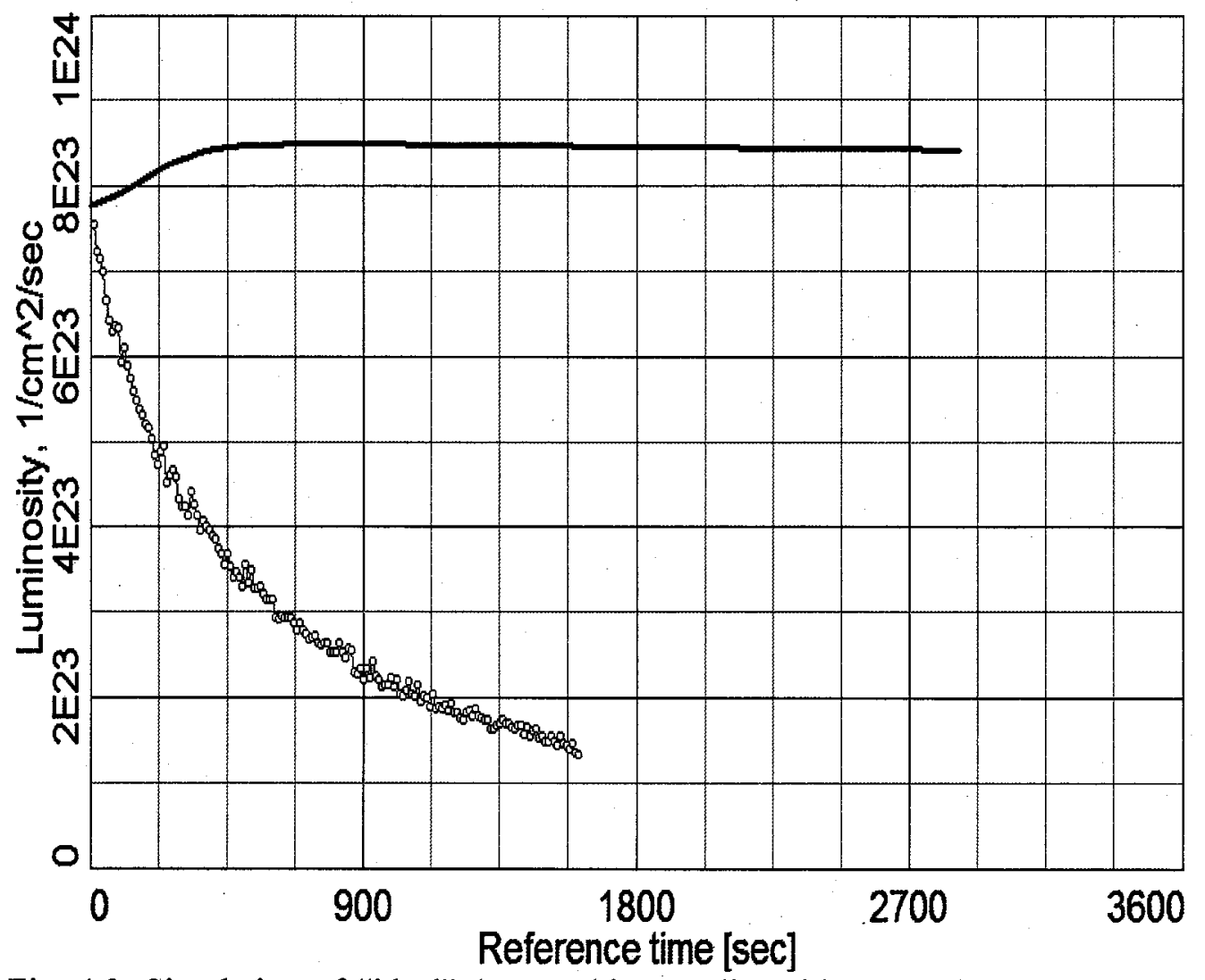

Fig. 4.3. Simulation of "ideal" (no machine nonlinearities were included, just IBS and beam loss from RF bucket) luminosity with (blue line) and without (black dots) electron cooling at $\gamma=2.7$ for 56 bunches.

Figure 4.4 shows a simulation of "ideal" luminosity performance with and without electron cooling for $\gamma=6.6$. Simulations are done for ion bunch intensity $\mathrm{N}_{i}=1 \times 10^{9}$, initial $95 \%$ normalized emittance of $15 \mathrm{~mm} \mathrm{mrad}, \sigma_{\mathrm{p}}=5 \times 10^{-4}, \sigma_{\mathrm{s}}=1.9 \mathrm{~m}$. For these parameters we are not yet space-charge limited. In such a case, in addition to just counteracting IBS, electron cooling allows to cool the transverse 
emittance to the space-charge limit, which in turn allows to decrease $\beta^{*}$ at the IP as can be seen by luminosity jump in the Fig. 4.4. Then the luminosity remains constant. For the scenario shown in Fig. 4.4, electron cooling provides about factor of 6 improvement in average luminosity.

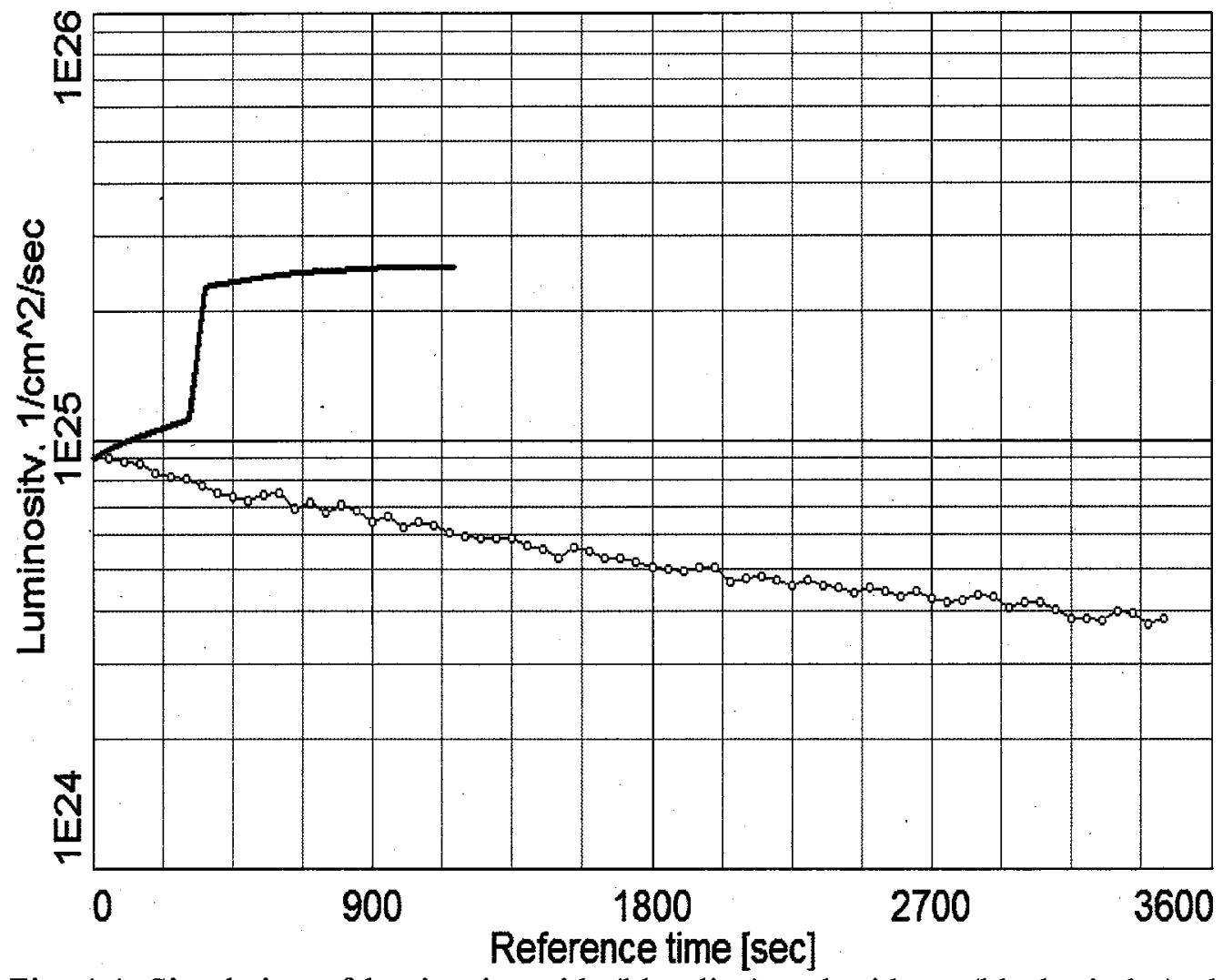

Fig. 4.4. Simulation of luminosity with (blue line) and without (black circles) electron cooling at $\gamma=6.6$.

For low-energy RHIC operation, maximum achievable luminosity will be limited by space-charge tune shift. One can significantly reduce the space-charge tune shift by providing collisions with coasting beams. In such a case, additional strong luminosity increase would be possible with electron cooling. This option is being discussed. However, since present timing system in RHIC detectors requires bunched ion beams, the standard scenario with bunched ion beams is presently assumed as a baseline approach.

To summarize, for typical colliding scenario with bunched beams, improvement due to electron cooling is limited by a space-charge limit in a collider. Electron cooling offers long stores for physics with about factor of 3 improvement (additional factor might be possible if one could operate with slightly larger tune shifts with the help of cooling) in average luminosity for low-energy points $(\gamma=2.7-5)$ and about factor of 5-6 improvement for higher energy points $(\gamma>5)$. Further improvement in luminosity may be possible if one considers collisions of coasting beams. 


\section{CONSIDERATIONS FOR ELECTRON COOLER}

The lowest energy points (ion kinetic energies $\mathrm{E}_{\mathrm{k}, \mathrm{i}}=1.6,2.2,2.9,3.45,5.2 \mathrm{GeV} /$ nucleon) can benefit the most from electron cooling; these correspond to electron beam kinetic energies $\mathrm{E}_{\mathrm{k}, \mathrm{e}}=0.9-2.8$ $\mathrm{MeV}$. Electron cooling at these energies was successfully demonstrated at Fermilab. Below we summarize several approaches to cooler design which may work for RHIC low-energy cooling.

\subsection{DC cooler}

Electron cooling with electron beam kinetic energies $\mathrm{E}_{\mathrm{k}, \mathrm{e}}=0.9-3 \mathrm{MeV}$ can be performed using a DC electron beam, as is being done in the Recycler cooler at Fermilab [12]. The Recycler cooler can operate in a wide energy range up to $5 \mathrm{MeV}$ electron kinetic energy. RHIC cooling times would be much smaller than those measured at the Recycler since we need to cool $\mathrm{Au}$ ions compared to antiprotons in the Recycler. The cooling time is thus reduced by a factor of $Z^{2} / A=31.7$, where $A=197$ and $Z=79$ are the atomic mass and charge of $A u$ ions, respectively.

However, some modification to the Recycler cooler will be needed in order to use it for RHIC. First, one would need to do some modification to the electron beam transport for low energies of electron beam, as well as evaluate the need of magnetic field during the initial stage of acceleration of the electron beam inside the Pelletron. Second, one will need to do modifications to the cooling section to compensate loss of heavy ions on recombination with electrons. Two approaches to combat recombination are being considered and are summarized in Section 5.4.

\subsection{RF cooler based on $56 \mathrm{MHz}$ SRF gun}

The energies of electron beam needed for low-energy RHIC are sufficiently high, which allows us to consider cooling using bunched electron beam. The main problem for bunched electron beam is to provide beam transport of electron bunches without significant degradation of beam emittance and energy spread. Using low-frequency RF gun provides very long electron bunches. As a result, even for high bunch charges, space-charge effects in such a bunch can be minimized. One can then deliver an electron beam of the necessary quality to the cooling section.

Preliminary simulations show that the required electron beam parameters can be obtained (see Appendix A.3 for details). The $56 \mathrm{MHz}$ gun is presently under design under SBIR by Niowave Inc. in Michigan and may be available for low-energy RHIC cooler in the future.

\subsection{RF cooler based on $703 \mathrm{MHz}$ SRF gun}

Presently, a $1 / 2$ cell $703.75 \mathrm{MHz}$ SRF gun is being built for the R\&D ERL at BNL [13]. It is thus an attractive scenario to use this gun later in the RHIC tunnel. The length of electron bunches at this frequency is very short (about $1 \mathrm{~cm} \mathrm{rms)} \mathrm{which} \mathrm{would} \mathrm{result} \mathrm{in} \mathrm{quick} \mathrm{increase} \mathrm{of} \mathrm{momentum} \mathrm{spread}$ of electron beam due to the longitudinal space charge. However, the length of ion beam is very large with 1.9 meters rms. This allows us to put about 20 electron bunches on a single ion bunch. The 
charge needed for cooling can be thus divided between 20 electron bunches resulting in 50pC per bunch. With such a low charge electron beam emittance is very small and is not an issue. The energy spread due to longitudinal space charge is also greatly reduced. The cooling in such a scenario is provided by a pulse/train of bunches with 20 bunches in the train spaced by $42.6 \mathrm{~cm}$ apart. The repetition frequency of such bunch trains is $9.38 \mathrm{MHz}$.

Preliminary simulations show that it should be possible to maintain required energy spread at the level of $5 \times 10^{-4}$ through the cooling section for $50 \mathrm{pC}$ bunch. This requires a special set-up which is described in Appendix A.4. Also, with such an approach, the resulting transverse angular spread satisfies cooling requirements as well. Simulations shown in Appendix A.4 indicate that the quality of the electron beam can be maintained at the required level through both of the cooling sections in Yellow and Blue RHIC rings, which makes this approach very attractive.

\subsection{Non-magnetized vs. magnetized approach}

In the cooling section, the interaction of the ion and electron beams results in ion beam loss due to recombination. In a standard electron cooler, recombination loss is minimized by incorporating a large transverse temperature of the electron beam. Subsequently, employment of s strong magnetic field in the cooling section allows one to remove the effect of the large transverse temperature of the electron beam from the cooling dynamics process. Such an approach is typically called "magnetized" cooling.

One the other hand, a novel idea of suppression ion recombination based on the use of an undulator field in the cooling section was proposed for RHIC [14]. In the presence of an undulator field, the trajectories of all the electrons have the same coherent azimuthal angle $\theta$, determined by the undulator period $\lambda$ and field value $B$ at the axis:

$$
\theta=\frac{e B \lambda}{2 \pi p c},
$$

where $p$ is the electron momentum. Since the recombination cross section is approximately inversely proportional to the electron energy in the ion rest frame, the ion beam lifetime can be sufficiently improved.

Using an undulator to suppress recombination would allow one to use non-magnetized electron beam with relatively small temperatures for cooling. To make sure that the representation of the friction force in the presence of an undulator field is accurate, an undulator field was implemented in the VORPAL code [15], and systematic numerical simulations were performed for different strength of the magnetic field $B$ and pitch period $\lambda[16]$. Based on these studies the design of the high-energy electron cooler for RHIC was changed from magnetized cooling to non-magnetized cooling which significantly simplified electron beam transport and reduced the cost of the cooler [17-19].

For the low-energy cooler in RHIC both approaches of magnetized and non-magnetized cooling were considered. As for the case of high-energy RHIC-II cooler, it was shown that one can use a rather weak undulator with a magnetic field of about $3-5 \mathrm{G}(8 \mathrm{~cm}$ period) to combat recombination 
in the cooling section, which makes use of the non-magnetized cooling attractive for low-energy RHIC operation as well. This approach is presently our baseline approach.

Alternatively, one can consider conventional magnetized cooling approach for low-energy RHIC. For example, such a magnetized cooler design with $0.2 \mathrm{~T}$ magnetic field in the cooling section is chosen as a baseline for future HESR DC electron beam cooler at GSI [20]. For the RHIC case, the magnetized approach require cooling sections ( $10 \mathrm{~m}$ for each of the two RHIC rings) to be covered with solenoids with magnetic field of about $0.5 \mathrm{~T}$. The requirement on straightness of magnetic field lines in solenoids is about $5 \times 10^{-5}$. However, due to large magnetization such a scenario is probably not compatible with the SRF gun approach or the Recycler cooler (without serious and expensive modifications), thus leaving us just with the non-magnetized method for present consideration.

\subsection{Pros and Cons of different approaches.}

\subsubsection{DC approach}

\section{Advantages:}

1. Parameters of electron beam needed for cooling are achievable, as demonstrated experimentally at the Recycler cooler in FNAL.

2. The Recycler cooler (all hardware) may be available for free after Tevatron shutdown.

\section{Disadvantages:}

1. A very massive structure, which requires a lot of space.

2. Modifications to the Recycler cooler will be required to address the questions of recombination and electron beam transport at low energies.

\subsubsection{RF 56MHz approach}

\section{Advantages:}

1. Parameters of electron beam needed were achieved in simulations through the first cooling section.

2. The gun is presently under design by Niowave Inc. in Michigan.

3. This gun can be used together with R\&D ERL for other cooling applications in RHIC.

4. More compact than DC approach.

\section{Disadvantages:}

1. Electron beam parameters needed are achieved, so far, only in simulations.

2. If one wants to use the same electron beam for two cooling sections (to minimize cost) it becomes challenging to control beam emittance during 180 degrees bend.

3. It will take some time before such a gun will be built. It is not clear what parameters will be demonstrated.

4. The overall timeline may be not consistent with the timeline needed for low-energy RHIC program. 


\subsubsection{RF 703MHz approach}

Advantages:

1. The gun is presently being built for R\&D ERL at BNL.

2. It will minimize cost of moving of the full R\&D ERL into the RHIC tunnel for demonstration of Coherent Electron Cooling (proposed for eRHIC) in the future.

3. Much more compact than DC approach.

4. Overall timeline (commissioning of R\&D ERL in 2009) seems consistent with the timeline needed for low-energy RHIC cooling.

5. Parameters needed for cooling were demonstrated in simulations through both cooling sections in two RHIC rings.

Disadvantages:

1. It is not clear what parameters will be demonstrated after the gun is built.

2. Laser system becomes more complicated to provide pulses of electron bunches required.

\section{COST ESTIMATE}

Some preliminary cost estimate of electron cooler was performed both for the $\mathrm{DC}$ and $\mathrm{RF}$ electron beam approach. It suggests that the cost of such a cooler for Low-Energy RHIC operation should be under $\$ 5 \mathrm{M}$. Please note that the cost estimates listed below were done for planning purpose only.

1. Pelletron (DC) electron cooler from FNAL. This scenario assumes that all the hardware from the Recycler cooler (Pelletron, all magnets, instrumentation, controls) will be available and come for free.

1.1) Transportation (30+ton): $0.03 \mathrm{M}$

1.2) Using IR 2 Bldg., requires pit for Pelletron: $0.05 \mathrm{M}$

(Installation in 2:00 IR; IR is 27' tall. There is an available service building for the gas, water and other systems, along with about $3 \mathrm{MW}$ of available power)

1.3) Shielding, removable roof, heat, other: $0.5 \mathrm{M}$

1.4) Additional magnets (if needed), undulators: $0.5 \mathrm{M}$

1.5) Vacuum system: $0.6 \mathrm{M}$

1.6) Non C-AD installation/labor: $2.0 \mathrm{M}$

Total for DC approach: $\$ 3.7 \mathrm{M}$

\section{RF cooler based $1 / 2$ cell SRF gun from R\&D ERL in Bldg. 912:}

2.1) Water cooling system (for driver \& He compressor skid): $0.05 \mathrm{M}$

2.2) Laser/Cryogenics Bldg.: $0.25 \mathrm{M}$

2.3) Power additions: $0.15 \mathrm{M}$ 
2.4) Transport magnets, power supplies: $0.75 \mathrm{M}$

2.5) Cooling section shielding, magnets, undulators: $0.5 \mathrm{M}$

2.6) Vacuum system: $0.6 \mathrm{M}$

2.7) Refrigerated LHe system: $0.5 \mathrm{M}$

2.8) Instrumentation/controls: $0.4 \mathrm{M}$

2.9) Cryogenics: $0.3 \mathrm{M}$

2.10) Energy correction cavity: $0.1 \mathrm{M}$

2.11) Non C-AD installation/labor: $1.0 \mathrm{M}$

Total for RF approach: $\$ 4.6 \mathrm{M}$

The third option based on $56 \mathrm{MHz}$ SRF gun should be comparable in cost to option 2, assuming that the gun with the cryostat will be available for BNL for free.

The largest contribution to cost estimate is labor. Presently, without detailed design, this item also has the largest uncertainty.

In addition to cost an important consideration for choosing one of the available approaches for the cooler is the timeline. For example, to provide Au ions collisions during Run-12, would require installation and commissioning of the cooler in 2011, which would require Technical Design of the cooler to start in 2008. In general, it is estimated that a needed minimum time from the start of Technical Design to construction and commissioning of such a cooler is about 3 years.

\section{ACKNOWLEDGMENTS}

We would like to thank T. Roser, W. Fischer and other members of Collider-Accelerator department at BNL for many useful discussions and suggestions. We also thank A. Sidorin (JINR, Dubna) and S. Nagaitsev, A. Shemyakin, L. Prost (FNAL) for providing useful information. We are grateful to Al Pendzick for providing cost estimate.

\section{REFERENCES}

[1] Proc. of Workshop "Can we discover QCD critical point at RHIC?" (BNL, March 2006) RIKEN BNL Research Center Report No. BNL-75692-2006; http://www..bnl.gov/riken/QCDRhic.

[2] A. Cho, Science, V. 312, April 12, 2006, p 190.

[3] G. Stephans, "critRHIC: the RHIC low energy program", J. Phys. G: Nucl. Part. Phys. 32 (2006).

[4] M. Stephanov, K. Rajagopal, and E. Shuryak, Phys. Rev. Letters 81, p. 4816 (1998).

[5] T. Satogata et al., "RHIC challenges for low energy operation", Proceed. of PAC07 (Albuquerque, NM, 2007), p. 1877; T. Satogata et al., 2008 RHIC retreat, March 31, 2008.

[6] G. I. Budker, At. Energ. 22, p. 346 (1967). 
[7] BETACOOL code: http://lepta.jinr.ru; A. Sidorin et al., NIM A 558, p. 325 (2006).

[8] A. Fedotov, presentation in Ref. [1].

[9] A. Fedotov, I. Ben-Zvi, X. Chang, D. Kayran, T. Satogata, Proc. of COOL07 (Germany, 2007).

[10] H. Burkhardt et al., Proc. of EPAC04, p. 1846 (2004).

[11] J. Bosser et al., Nuc. Instr. Meth. A 441, p.1 (2000).

[12] S. Nagaitsev et al., Phys. Rev. Letters 96, 044801 (2006).

[13] V. N. Litvinenko et al., "Status of R\&D ERL at BNL", Proc. of PAC07, p. 1347.

[14] Ya. Derbenev, "Electron cooling in solenoid with undulator", TJLAB Note 2001, unpublished.

[15] C. Nieter, J. Cary, J. Comp. Phys. 196 , p.448 (2004); http://www.txcorp.com.

[16] G. Bell et al., Proc. of PAC07 (Albuquerque, NM, 2007), p. 3549.

[17] A. Fedotov et al., Proc. of PAC05 (Knoxville, TN, 2005), p. 4236.

[18] A. Fedotov et al., Proc. of PAC07 (Albuquerque, NM, 2007), p. 3696.

[19] Electron cooling for RHIC: http://www.bnl.gov/cad/ecooling

[20] B. Gålnander et al., Proc. of COOL07 (Bad Kreuznach, Germany), p.182 (2007).

\section{APPENDIX}

\section{A.1 List of parameters}

In this section we provide a short list of main parameters for electron cooler.

\section{Ion beam Parameters:}

Gold ions kinetic energies for cooling: 1.6-5.2 GeV/nucleon

Ion number per bunch: $0.5-1 \times 10^{9}$

Ion charge: 79

Initial ion normalized rms emittance: about $2.5 \mu \mathrm{m}$

(in both transverse planes)

Initial rms momentum spread: $5 \cdot 10^{-4}$

$\mathrm{RF}$ frequency: about $28 \mathrm{MHz}$

Bunch frequency: $9.38 \mathrm{MHz}$

$\mathrm{RF}$ voltage: up to $500 \mathrm{kV}$ per ring

\section{Cooling section and electron beam:}

Undulator, helical, length: 10 meter (for each ring)

Undulator magnetic field range: $3-5 \mathrm{G}$

Undulator period: $8 \mathrm{~cm}$

Electron rms beam size in cooling section: $1 \mathrm{~cm}$ (for lowest energy)

Kinetic energy $0.87-2.8 \mathrm{MeV}$

Total bunch charge (RF beam): $1-2 \mathrm{nC}$

rms normalized emittance (for $1 \mathrm{nC}$ ) $\leq 3 \mu \mathrm{m}$

rms normalized emittance (for $2 \mathrm{nC}$ ) $<6 \mu \mathrm{m}$

rms momentum spread: $\leq 0.0005$ 


\section{A.2 Parameters of DC electron beam}

At low energy RHIC ion bunches are very long (rms bunch length 1.6-1.9 m) with the full bunch length up to $30 \mathrm{nsec}$. DC electron beam is ideally suited for cooling of such long ion bunches. To produce an effective charge of $1 \mathrm{nC}$ (needed to just counteract IBS for lowest energy point) requires only $0.033 \mathrm{~A}$ of DC current. To provide also some additional cooling of beam emittance for high energy points overall requirement on electron beam current is $0.03-0.1 \mathrm{~A}$.

Good performance of electron cooler with currents up to $0.5 \mathrm{~A}$ was demonstrated at the Recycler cooler in FNAL. The Recycler cooler operates at $4.3 \mathrm{MeV}$ kinetic energy of electrons. For lowenergy RHIC, we need to go as low as $0.87 \mathrm{MeV}$. Transport of electron beam at such low energy and resulting electron beam parameters must be studied carefully.

\section{A.3 Electron beam dynamics for $56 \mathrm{MHz} R \mathrm{R}$ cooler}

This section summarizes electron beam dynamics studies for $56 \mathrm{MHz}$ SRF gun approach obtained with PARMELA code.

Figure A.3.1 shows simplified schematic of the cooler (without bending of electron beam), which was used for beam dynamics studies initially.

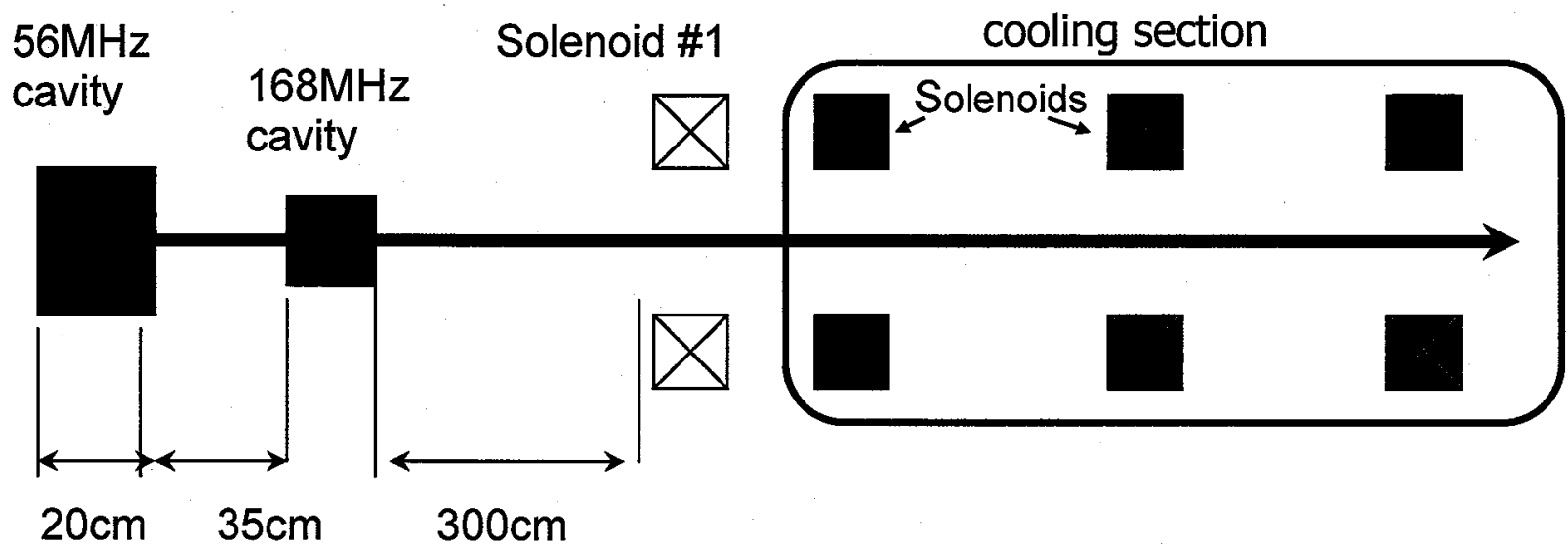

Fig. A.3.1 Schematic drawing of electron beam transport based on $56 \mathrm{MHz} \mathrm{RF}$ gun.

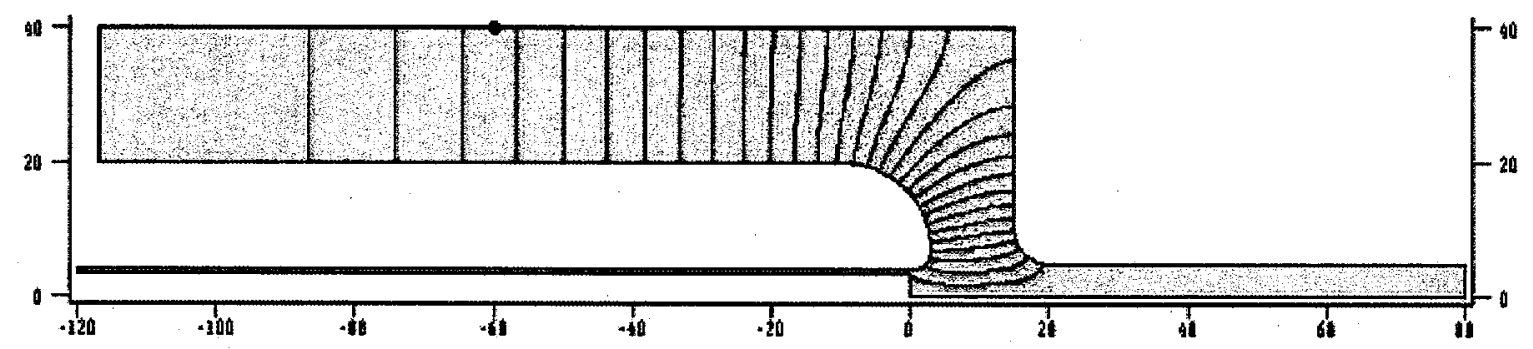

Fig. A.3.2. Fields of $56 \mathrm{MHz}$ RF gun. Peak field on axis is $11 \mathrm{MV} / \mathrm{m}$, Peak field on the wall is $18 \mathrm{MV} / \mathrm{m}$. 


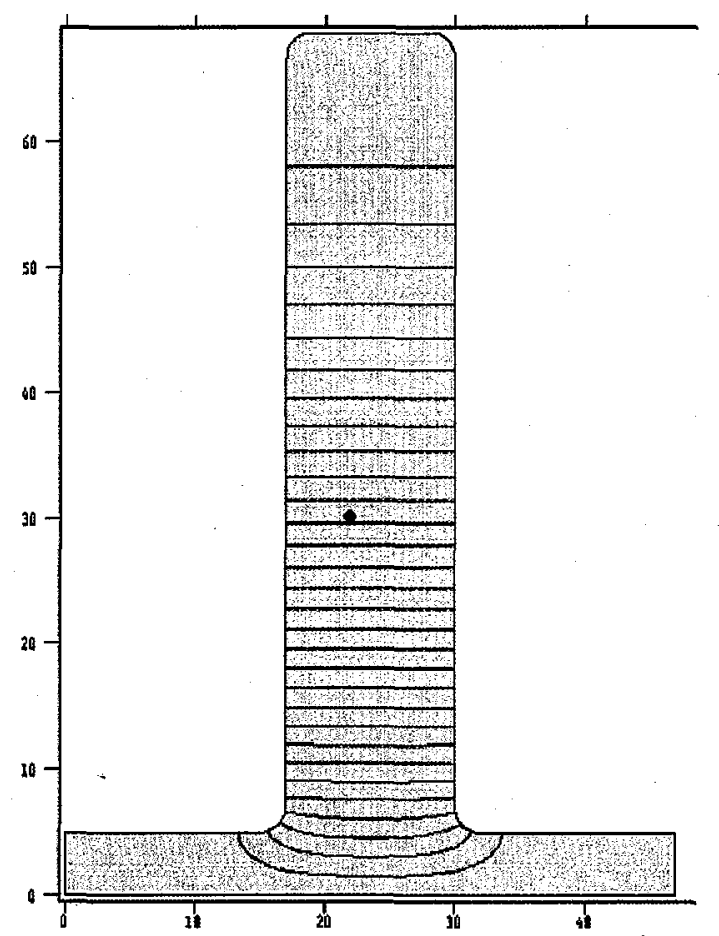

Fig. A.3.3. $3^{\text {rd }}$ harmonic cavity $(168 \mathrm{MHz})$. Peak field on axis is $1.25 \mathrm{MV} / \mathrm{m}$, Peak field on the wall is $1.8 \mathrm{MV} / \mathrm{m}$.

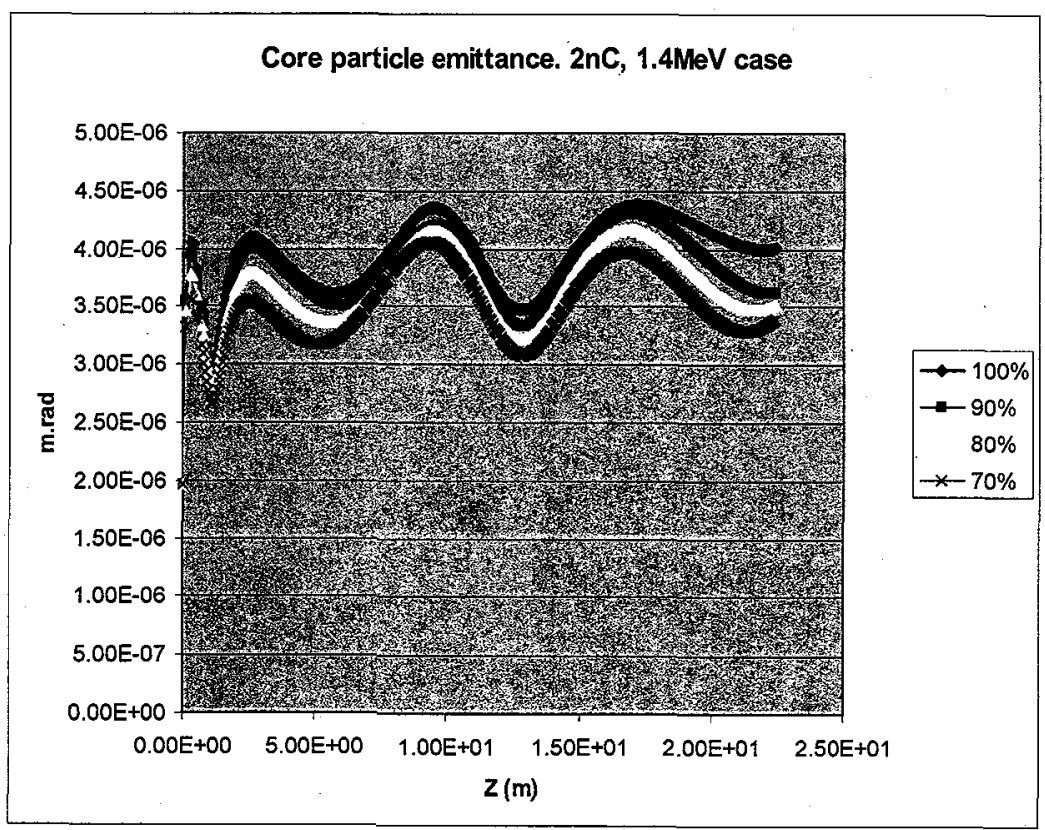

Fig. A.3.4. Time evolution of rms normalized emittance at electron kinetic energy of $E_{\mathrm{k}, \mathrm{e}}=1.4 \mathrm{MeV}$. 


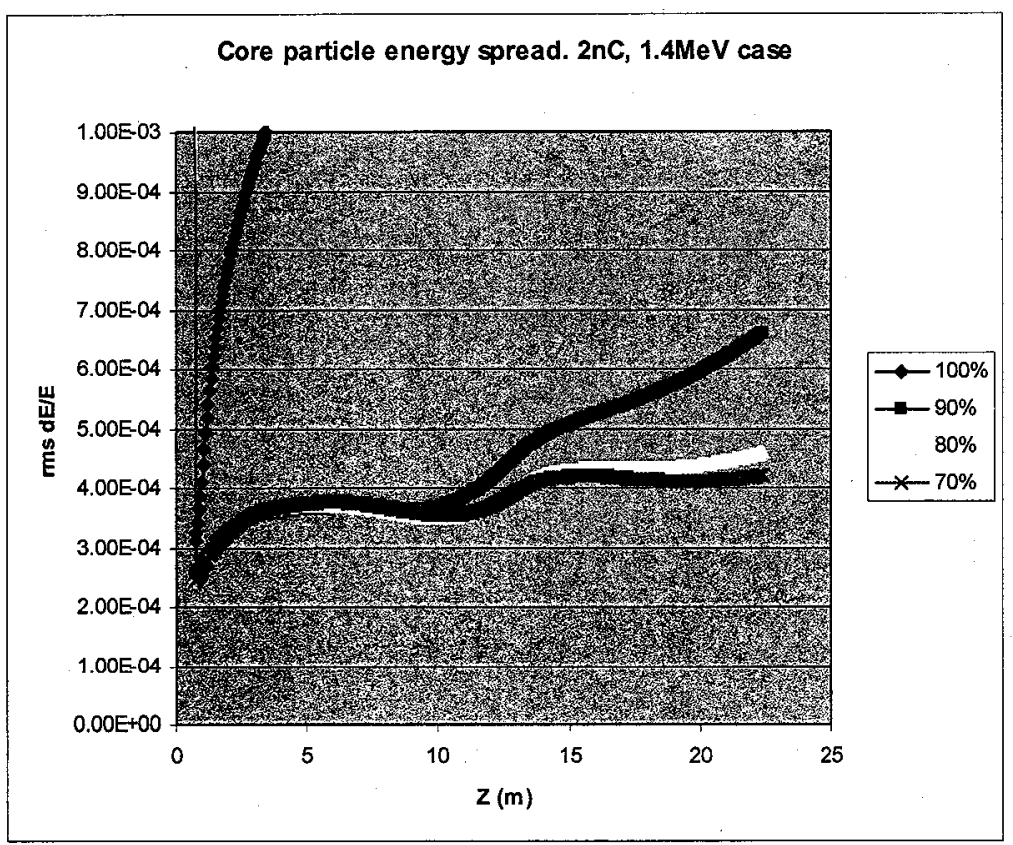

Fig. A.3.5. Time evolution of rms energy spread at electron kinetic energy of $E_{k, e}=1.4 \mathrm{MeV}$.

More realistic electron beam transport should include bending magnet to merge electron beam with the ion beam. An example of such system is shown in Fig. A.3.6.

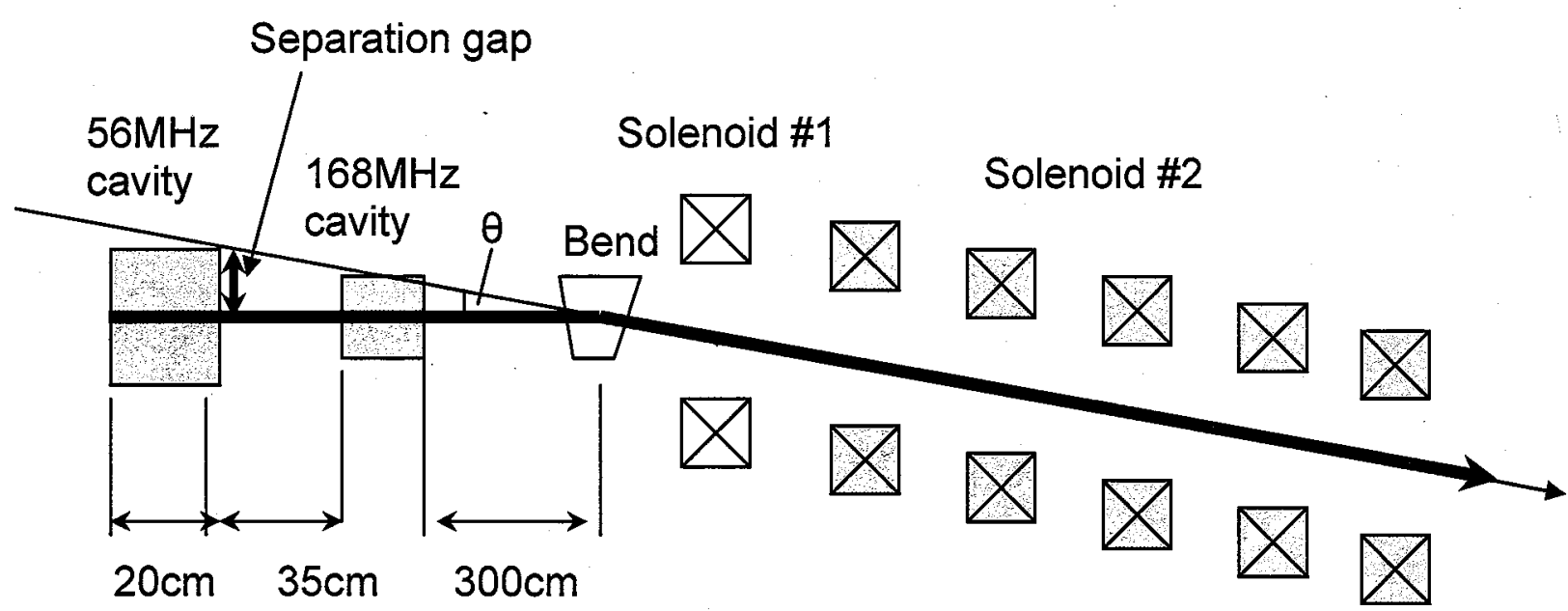

Fig. A.3.6. Schematic drawing of electron beam transport with the merge. Direction of electron beam is shown with green line while direction of ion beam is shown with straight black line.

A merging angle of 5 degrees corresponds to $29 \mathrm{~cm}$ separation gap between electron and ion beam lines; 10 degrees corresponds to $58 \mathrm{~cm}$, and 15 degrees corresponds to $87 \mathrm{~cm}$. Simulations shown in Figs. A.3.7-9 were done for a 15 degrees merging angle.

For the low-energy point with kinetic energy of $0.85 \mathrm{MeV}$ it is difficult to preserve the momentum spread at small level even for a very long bunch (45cm full length in this case), as shown in Fig. A.3.7. However, for such a long bunch one would expect that longitudinal space charge is affectively screened by particles at distances larger than the beam-pipe radius. Simulations which 
include beam-pipe wall and thus account for such screening effect are shown in Fig. A.3.8. One can see that growth of momentum spread is decreased due to the screening effect.

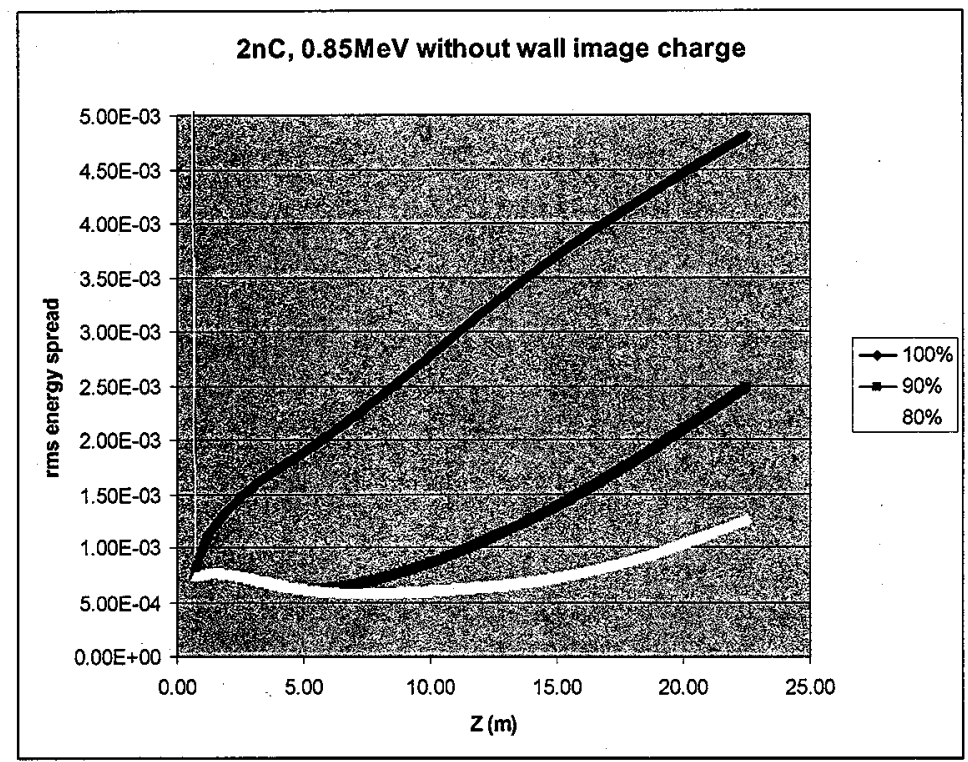

Fig. A.3.7. Time evolution of rms energy spread at electron kinetic energy of $E_{k, e}=0.85 \mathrm{MeV}$ without screening due to wall images.

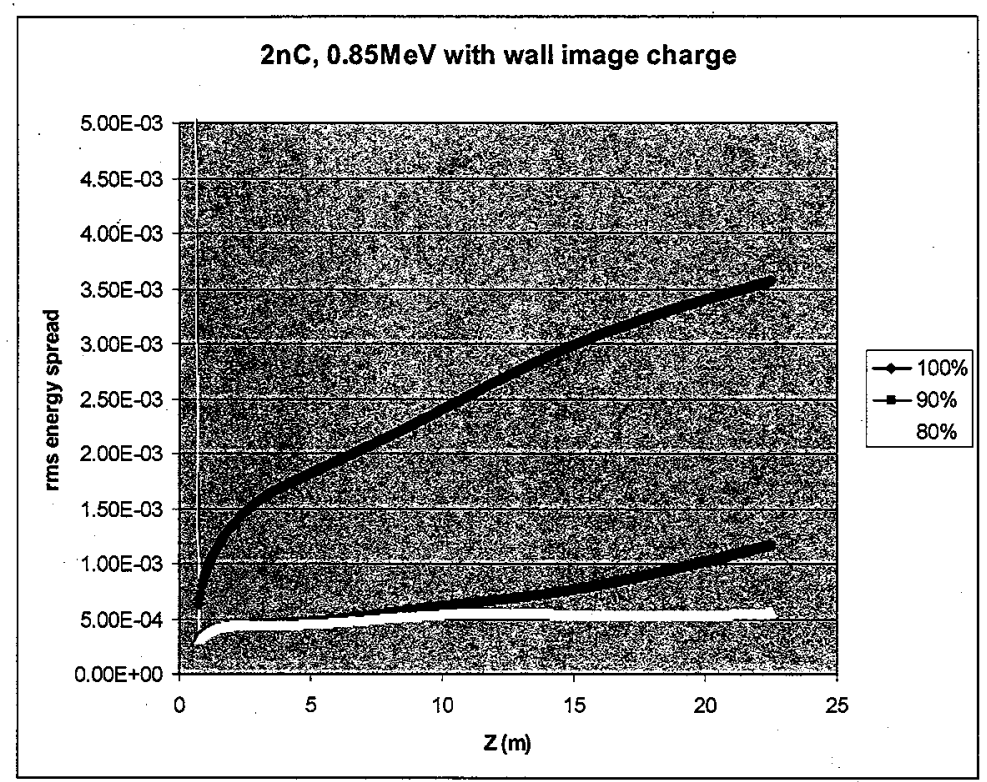

Fig. A.3.8. Time evolution of rms energy spread at $E_{k, e}=0.85 \mathrm{MeV}$ with screening due to wall images.

Figure A.3.8 shows that $80 \%$ of $2 \mathrm{nC}$ beam has rms energy spread which satisfies requirement of $5 \times 10^{-4}$ needed for cooling. The effective charge (with good energy spread) is thus $1.6 \mathrm{nC}$, which also satisfies requirement of $\mathrm{InC}$ minimum. Corresponding rms normalized emittance, for the merging system in Fig. A.3.6, is shown in Fig. A.3.9. 


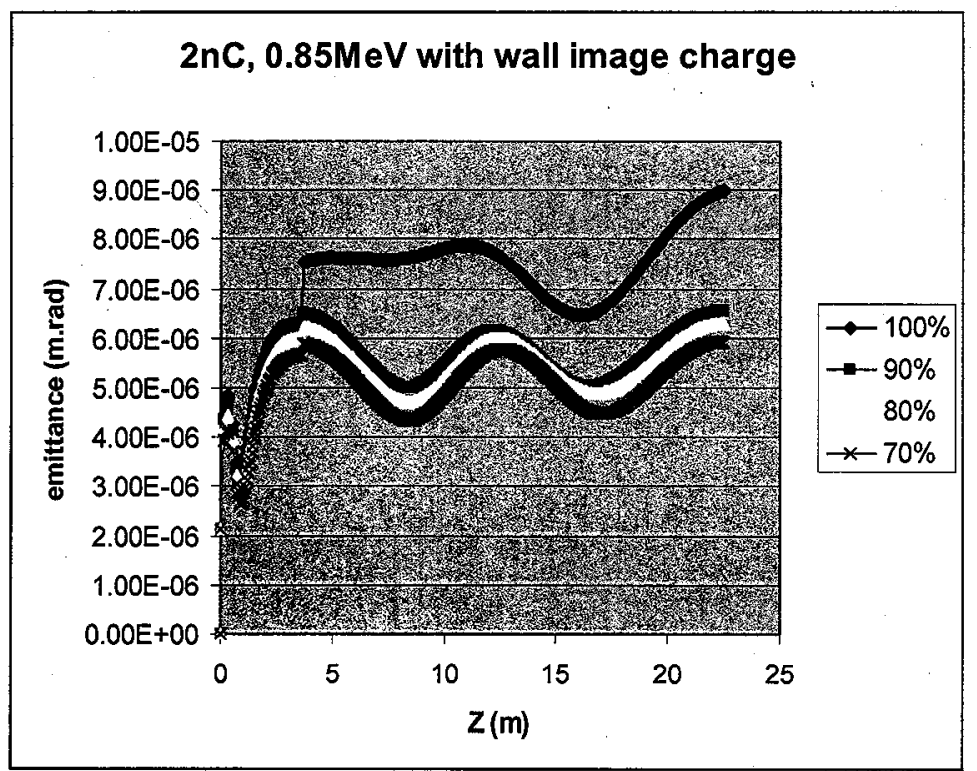

Fig. A.3.9. Time evolution of rms emittance at $\mathrm{E}_{\mathrm{k}, \mathrm{e}}=0.85 \mathrm{MeV}$.

Electron beam dynamics simulations presented in this section show that the parameters needed for cooling can be achieved for the beam transport through the cooling section in one RHIC ring. However, if one wants to minimize cooler's cost by using the same electron beam to provide cooling in two RHIC rings, this requires turning the electron beam after the first cooling section by 180 degrees and delivering a good quality beam to the second cooling section in the other RHIC ring. This presents a challenge for beam dynamics at such low energies. Feasibility of preserving electron beam parameters for second cooling section is presently under investigation.

\section{A.4 Electron beam dynamics for $703 \mathrm{MHz}$ RF cooler}

The frequency of the $1 / 2$ cell gun of R\&D ERL (under construction at BNL) is $703.75 \mathrm{MHz}$. For lowenergy cooling, one will need to operate this gun in CW mode with 10-20 mA of current up to 2.8 $\mathrm{MeV}$ kinetic energy beam.

The length of electron bunches at this frequency is very short (about $1 \mathrm{~cm} \mathrm{rms}$ in the lab frame) which results in quick increase of momentum spread of electron beam due to the longitudinal spacecharge. However, the length of ion beam is very large with 1.9 meters rms. This allows us to put about 20 electron bunches on a single ion bunch, which is schematically shown in Fig. A.4.1. The charge needed for cooling can be thus divided between 20 electrons bunches resulting in 50pC per bunch. 


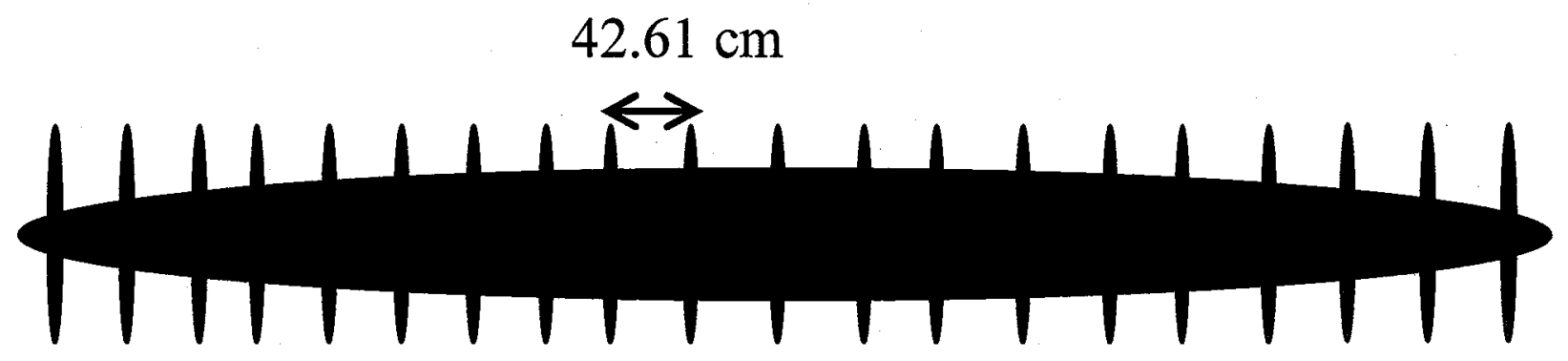

\section{Ion bunch length: full bucket $\sim 10 \mathrm{~m}$}

Fig. A.4.1. Schematic picture which shows train of equally spaced electron bunches (green disks) timed to a single ion bunch (blue ellipse) in RHIC.

Preliminary simulations suggest that it is hard to maintain required energy spread at the level of $5 \times 10^{-4}$ during the 10 meters of cooling section even for $50 \mathrm{pC}$ bunch if this bunch is as short as $1 \mathrm{~cm}$ rms. However, one can stretch electron bunches in the pulse train by accelerating at the linear slope of the RF. The resulting large energy spread is then corrected by an additional cavity of the same frequency, which is schematically shown in Fig. A.4.2.

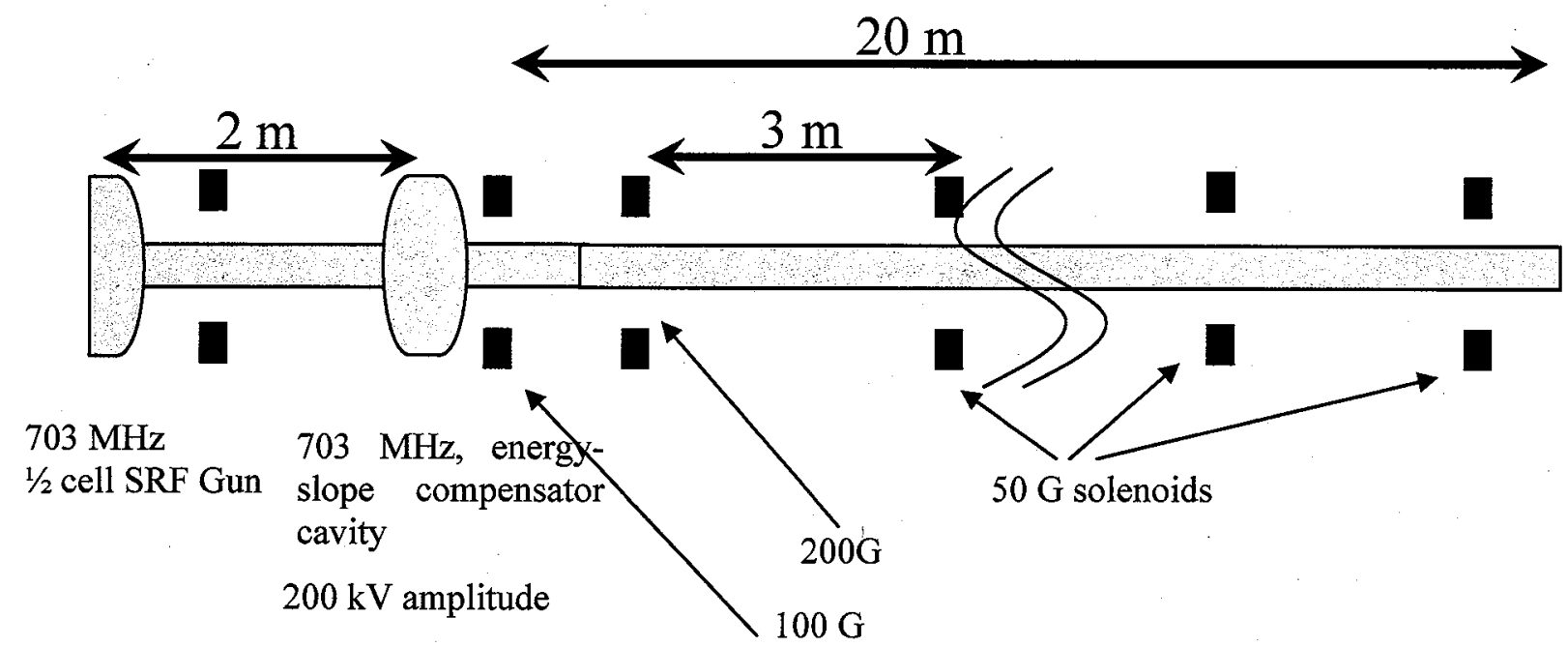

Fig. A.4.2. Schematic picture which shows set-up with stretched electron bunches and energy correction cavity. 


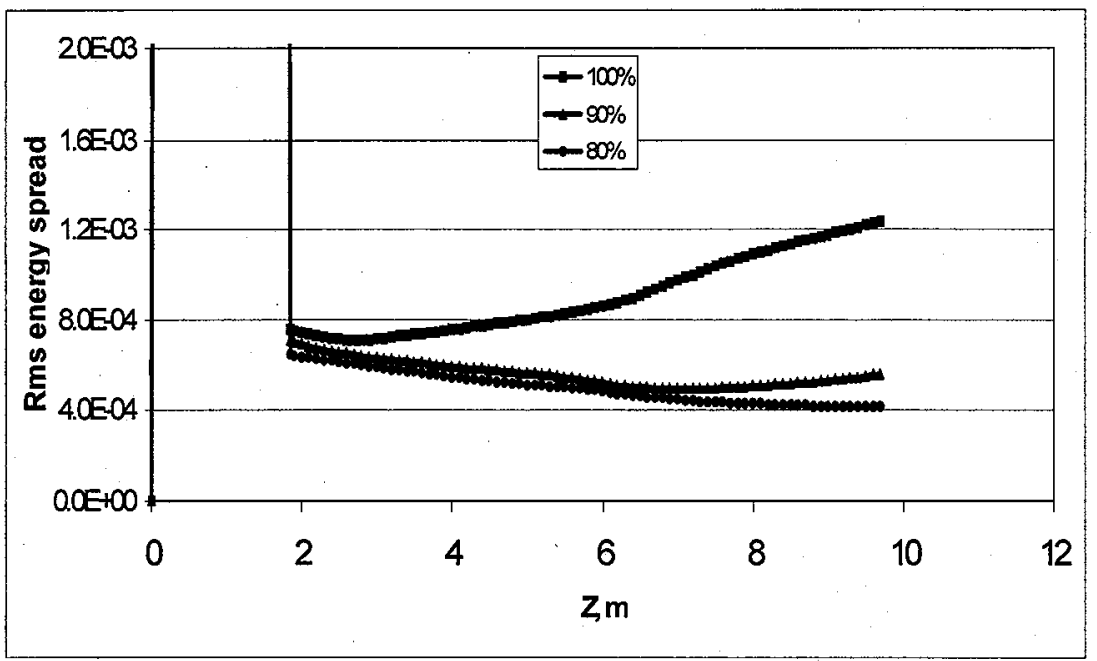

Fig. A.4.3. Time evolution of rms energy spread through the first cooling section for the set-up shown in Fig. A.4.2, for the lowest energy point with $\gamma=2.7$.

Simulations with the set-up in Fig. A.4.2 look very promising, with $90 \%$ of the beam satisfying requirement on energy spread from cooling.

Such a scenario with $50 \mathrm{pC}$ per bunch also allows us to maintain sastifactory emittance (tranverse angular spread) of electron beam including turn around of electron beam after the first cooling section so that electron beam could be used one more time for cooling of ions in the other RHIC ring. Preliminary results of simulations through both cooling sections (10 meters in Yellow ring and 10 meters in Blue ring), including turning electron beam around, are shown in Figs. A.4.4 and A.4.5 for the transverse emittance and agular spread, respectively.

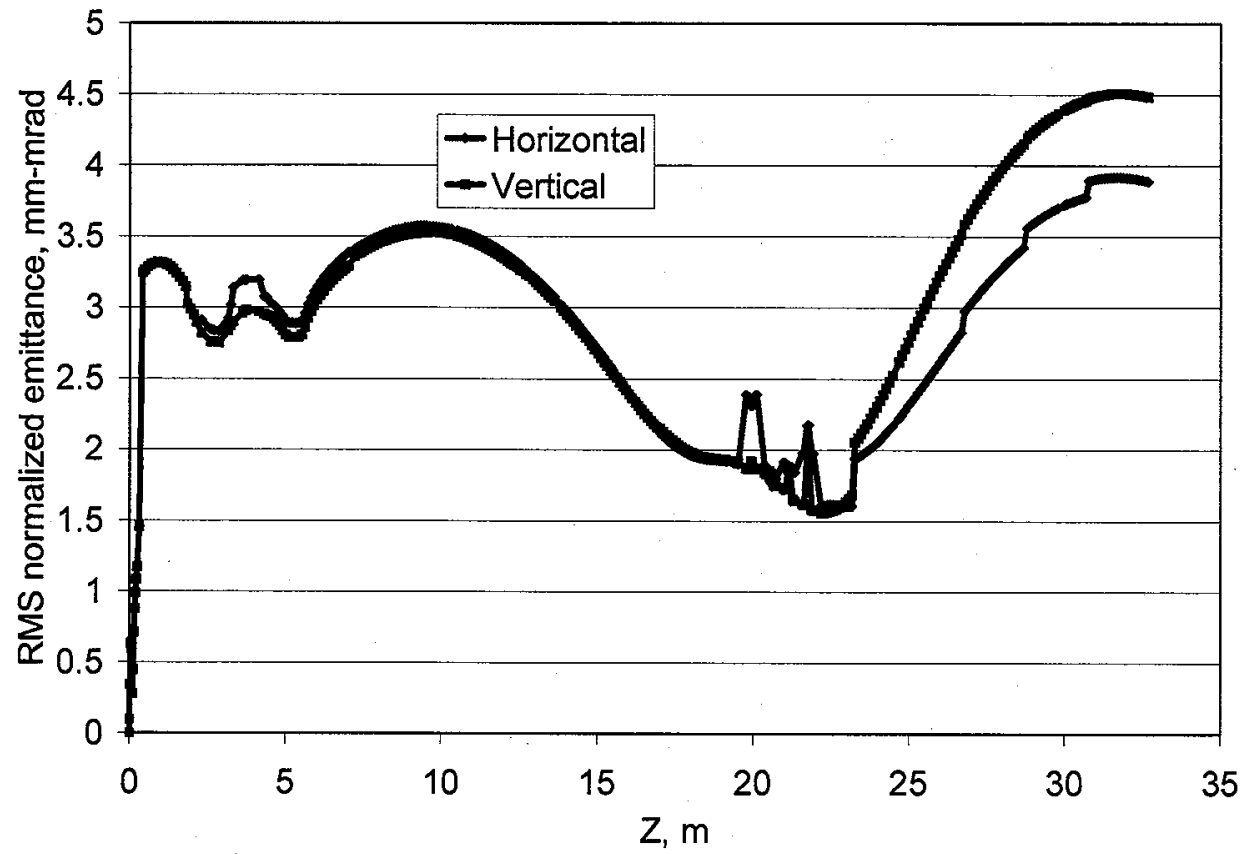

Fig. A.4.4. Time evolution of rms emittance of $50 \mathrm{pC}$ electron bunch in the bunch train, for the lowest energy point with $\gamma=2.7$. 


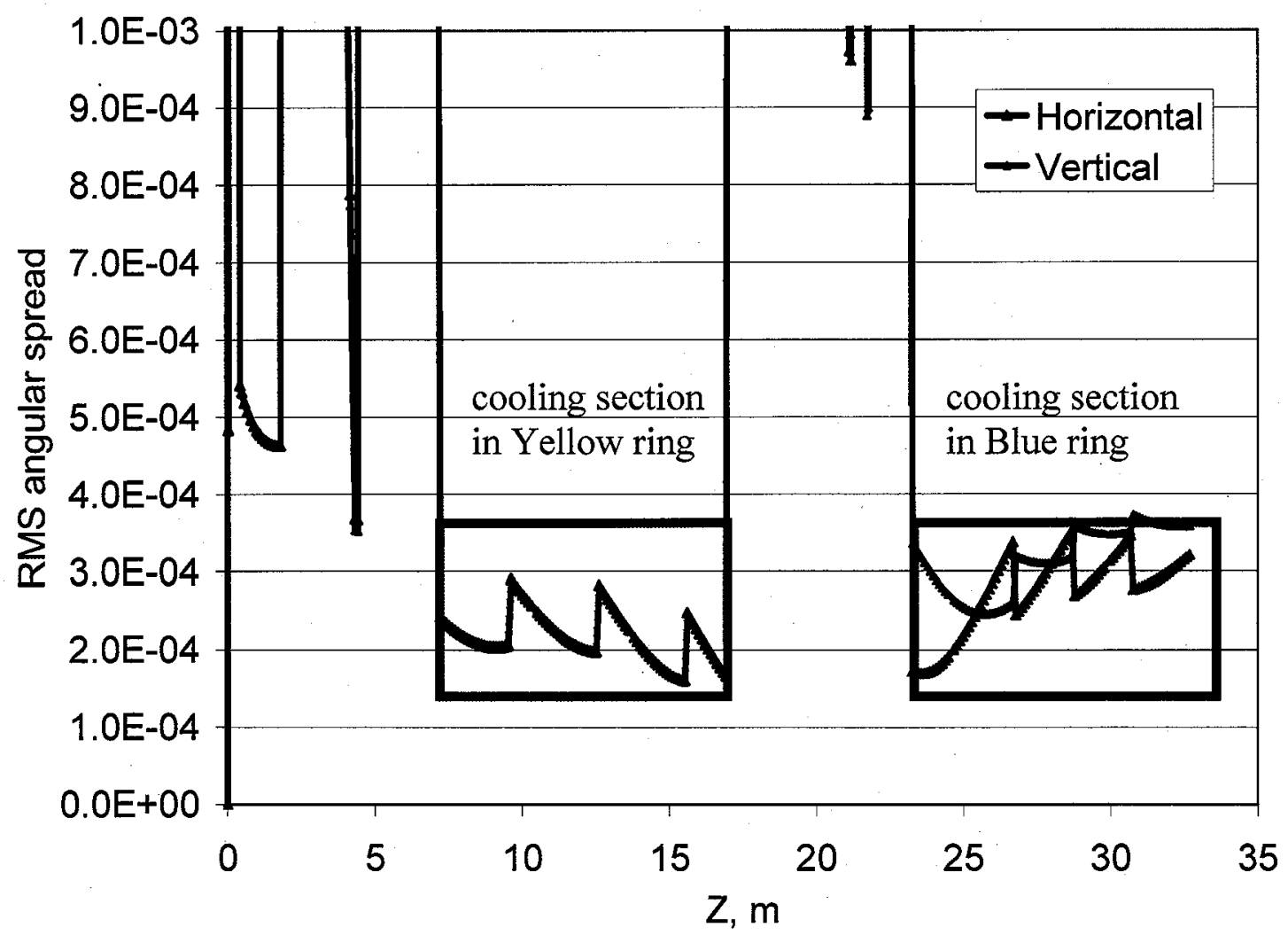

Fig. A.4.5. Time evolution of transverse rms angular spread through both cooling section in Yellow and Blue rings, for the lowest energy point with $\gamma=2.7$.

The strength of electron cooling strongly depends on the relative rms angular spread of electron and ion beams. For RHIC at low energies, the requirement on relative rms angular spread comes from ion energy spread which is about $0.0005 \mathrm{rms}$. As a result, the quality of electron beam should be such that both rms energy spread and transverse rms angular spread of electron beam should be comparable or smaller than 0.0005 . Figure A.4.5 shows that transverse rms angular spread of electron bunches could be controlled to an acceptable level through both of the cooling sections. 


\section{A.5 Cooling section.}

The cooling section is the region where the electron beam and ion beam overlap to produce the cooling action. The electron beam cools ions in RHIC Yellow ring then it is turned around and cools ions in RHIC Blue ring before going to the dump (in the RF electron beam approach). The electron beam must maintain its peak performance all along electron beam transport through the second cooling in Blue ring.

The Blue and Yellow ring cooling sections are about 11 meters each. For the non-magnetized approach most of cooling section is covered by helical undulators. The helical undulators in the cooling section are used to suppress recombination of the heaviest ions. For the magnetized approach, one would use strong solenoids instead of undulators to combat recombination. Some space is taken up by closely spaced steering dipoles and beam position monitors used to keep the electron beam and ion beam in close relative alignment. Pairs of weak solenoids are used in the nonmagnetized approach to compensate the effects of transverse space charge defocusing. The schematic layout of cooling section is shown in Fig. A.5.1.

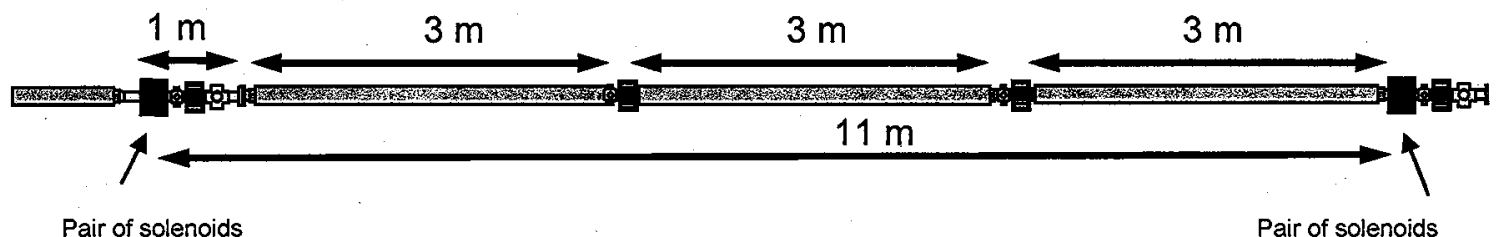

Fig. A.5.1. Schematic layout of $11 \mathrm{~m}$ cooling section consisting of $3 \times 3 \mathrm{~m}$ helical undulators, 3 steering dipoles, pair of solenoids, vacuum equipment and instrumentation line. 